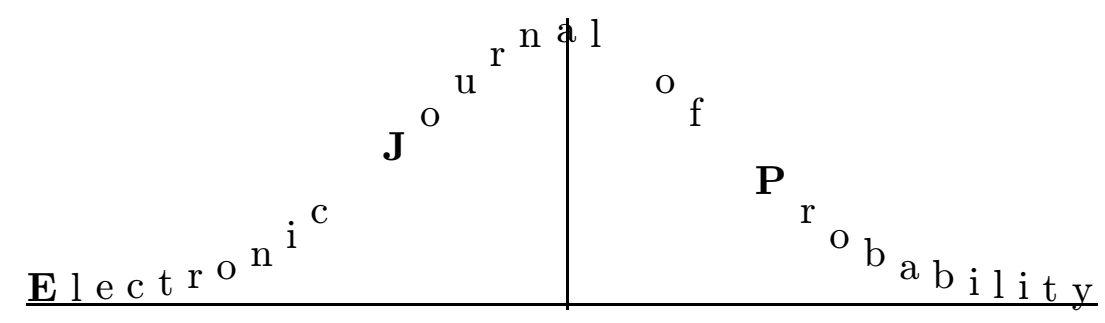

Vol. 3 (1998) Paper no. 4, pages 1-36.

Journal URL

http://www.math.washington.edu/ ejpecp/

Paper URL

http://www.math.washington.edu/ ejpecp/EjpVol3/paper4.abs.html

\title{
GEOMETRIC EVOLUTION UNDER ISOTROPIC STOCHASTIC FLOW
}

\author{
M. CRanston And Y. LeJan \\ University of Rochester \\ Rochester, New York, USA \\ cran@math.rochester.edu
Université de Paris, Sud
Orsay 91405, France \\ Yves.LeJan@math.u-psud.fr
}

\begin{abstract}
Consider an embedded hypersurface $M$ in $\mathbb{R}^{3}$. For $\Phi_{t}$ a stochastic flow of differomorphisms on $\mathbb{R}^{3}$ and $x \in M$, set $x_{t}=\Phi_{t}(x)$ and $M_{t}=\Phi_{t}(M)$. In this paper we will assume $\Phi_{t}$ is an isotropic (to be defined below) measure preserving flow and give an explicit descripton by SDE's of the evolution of the Gauss and mean curvatures, of $M_{t}$ at $x_{t}$. If $\lambda_{1}(t)$ and $\lambda_{2}(t)$ are the principal curvatures of $M_{t}$ at $x_{t}$ then the vector of mean curvature and Gauss curvature, $\left(\lambda_{1}(t)+\lambda_{2}(t), \lambda_{1}(t) \lambda_{2}(t)\right)$, is a recurrent diffusion. Neither curvature by itself is a diffusion. In a separate addendum we treat the case of $M$ an embedded codimension one submanifold of $\mathbb{R}^{n}$. In this case, there are $n-1$ principal curvatures $\lambda_{1}(t), \ldots, \lambda_{n-1}(t)$. If $P_{k}, k=1$, $n-1$ are the elementary symmetric polynomials in $\lambda_{1}, \ldots, \lambda_{n-1}$, then the vector $\left(P_{1}\left(\lambda_{1}(t), \ldots, \lambda_{n-1}(t)\right), \ldots, P_{n-1}\left(\lambda_{1}(t), \ldots, \lambda_{n-1}(t)\right)\right.$ is a diffusion and we compute the generator explicitly. Again no projection of this diffusion onto lower dimensions is a diffusion. Our geometric study of isotropic stochastic flows is a natural offshoot of earlier works by Baxendale and Harris (1986), LeJan (1985, 1991) and Harris (1981).
\end{abstract}

1991 Mathematics Subject Classification. 60H10, 60J60.

Key words and phrases. Stochastic flows, Lyapunov exponents, principal curvatures.

Research supported by NSA and Army Office of Research through MSI at Cornell. Submitted to EJP on February 19, 1996. Final version accepted on February 12, 1998. 


\section{$\S 1$ Isotropic Flows and Geometric Setting.}

We begin with a nonnegative measure $F(d \rho)$ on $[0,+\infty)$ with finite moments of all orders. The $p$ th moment of $F$ will be denoted by $\mu_{p}$. To $F$ we can associate a covariance

$$
C_{i j}(z)=\int_{0}^{\infty} \int_{S^{n-1}} e^{i \rho\langle z, t\rangle}\left(\delta_{j}^{i}-t^{i} t^{j}\right) \sigma_{n-1}(d t) F(d \rho),
$$

where $\sigma_{n-1}$ is normalized Lebesgue measure on $S^{n-1}$. By a result of Kolmogorov, to $C_{i j}$ is associated a smooth (in $x$ ) vector-field valued Brownian motion $U_{t}(x)$ such that

$$
E U_{t}^{i}(x) U_{s}^{j}(y)=(t \wedge s) C^{i j}(x-y), \quad 1 \leq i, j \leq n .
$$

A Brownian flow on $\mathbb{R}^{n}$ is constructed by solving the equation

$$
\Phi_{t}(x)=x+\int_{0}^{t} \partial U_{s}\left(\Phi_{s}(x)\right)
$$

( $\partial$ denotes Stratonovich differential.) Existence and uniqueness of such flows was proved in Baxendale (1984), LeJan and Watanabe (1984). Then if $T_{a}$ is translation by $a \in \mathbb{R}^{n}, T_{a} \Phi_{t} T_{-a}$ and $\Phi_{t}$ are identical in law so $\Phi$ is stationary. If $R$ is unitary, $R \Phi_{t} R^{-1}$ and $\Phi_{t}$ are identical in law. When these two properties hold we say $\Phi$ is isotropic. These properties both follow from the nature of $C_{i j}$ so our $\Phi$ is isotropic. The field $U_{t}(x)$ is smooth so we differentiate it writing

$$
\begin{gathered}
W_{j}^{i}=\frac{\partial}{\partial x^{j}} U^{i} \\
B_{j k}^{i}=\frac{\partial^{2}}{\partial x^{j} \partial x^{k}} U^{i} .
\end{gathered}
$$

Then we have as direct consequence of (1.1) and (1.2) (using $\langle$,$\rangle to denote both$ Euclidean inner product as well as quadratic variation for martingales, $d$ will denote the Itô differential.)

$$
\begin{gathered}
\left\langle d W_{j}^{i}(t, y), d W_{\ell}^{k}(t, y)\right\rangle=\frac{\mu_{2}}{n(n+2)}\left[(n+1) \delta_{k}^{i} \delta_{\ell}^{j}-\delta_{j}^{i} \delta_{\ell}^{k}-\delta_{\ell}^{i} \delta_{j}^{k}\right] d t \\
\left\langle d B_{j k}^{i}(t, y), d W_{q}^{p}(t, y)\right\rangle=0
\end{gathered}
$$

and for vectors $u, v \in \mathbb{R}^{n}$,

$$
\langle\langle d B(u, u), v\rangle,\langle d B(u, u), v\rangle\rangle=\frac{3 \mu_{4}}{n(n+2)(n+4)}\left[(n+3)\|u\|^{4}|| v\left\|^{2}-4\langle u, v\rangle^{2}\right\| u \|^{2}\right] d t
$$


By isotropy, (1.6), (1.7) and (1.8) remain valid under any unitary change of coordinates. We define,

$$
C_{j \ell}^{i k} d t=\left\langle d W_{j}^{i}, d W_{\ell}^{k}\right\rangle=\frac{\mu_{2}}{n(n+2)}\left[(n+1) \delta_{k}^{i} \delta_{\ell}^{j}-\delta_{j}^{i} \delta_{\ell}^{k}-\delta_{\ell}^{i} \delta_{j}^{k}\right] d t
$$

where both $d W_{j}^{i}, d W_{\ell}^{k}$ are evaluated at $(t, y)$, and

$$
C_{k \ell p q}^{i j} d t=\left\langle d B^{i}\left(e_{k}, e_{\ell}\right), d B^{j}\left(e_{p}, e_{q}\right)\right\rangle
$$

again both $d B$ terms are evaluated at $(t, y)$. A quick calculation shows $E\left(\sum_{i=1}^{n} d W_{i}^{i}\right)^{2}=0$ so $\operatorname{div} U=0$ and therefore $\Phi$ is a measure-preserving flow. Also, it's rather easy to check that $C_{1111}^{n n}=3 C_{1122}^{n n}$ and $C_{1122}^{n n}=\frac{(n+3) \mu_{4}}{n(n+2)(n+4)}$.

The most general form of $\left\langle d W_{j}^{i},(t, y), d W_{\ell}^{k}(t, y)\right\rangle=C_{j \ell}^{i k} d t$ for isotropic flows is $C_{j \ell}^{i k}=a \delta^{i, k} \delta_{j, \ell}+b \delta_{j}^{i} \delta_{\ell}^{k}+c \delta_{\ell}^{i} \delta_{j}^{k}$ where $a+c, a-c, a+c+d b$ are nonnegative (see LeJan (1985) and the references therein). We retrict our attention to the present covariance structure; $a=(n+1) \frac{\mu_{2}}{n(n+2)}, b=-\frac{\mu_{2}}{n(n+2)}, c=-\frac{\mu_{2}}{n(n+2)}$, as computations are massively simplified by the fact that certain Ito correction terms vanish. The works of Baxendale and Harris (1986) and LeJan (1985) focused on first order properties of the general isotropic flows in Euclidean space. That is, on properties of the derivative flow $D \Phi_{t}(x)$. Perhaps the most fundamental information about the derivative flow is its Lyapunov spectrum. To describe the Lyapunov spectrum, first extend $D \Phi_{t}$ to $p$-forms $\alpha=v_{1} \wedge \cdots \wedge v_{p}$ (we're in Euclidean space so we can identify forms and vectors) by defining $D \Phi_{t}(x) \alpha=D \Phi_{t}(x) v_{1} \wedge \cdots \wedge D \Phi_{t}(x) v_{p}$. Then if $v_{1}, \ldots, v_{p}$ are linearly independent, a.s.

$$
\lim _{t \rightarrow \infty} \frac{1}{t} \log \left\|D \Phi_{t}(x) \alpha\right\|=\gamma_{1}+\cdots+\gamma_{p}
$$

is the sum of the first $p$ Lyapunov exponents. In the case of the isotropic, measurepreserving flow on $\mathbb{R}^{n}, \gamma_{1}+\cdots+\gamma_{p}=\frac{n p(n-p) \mu_{2}}{2(n+2)}$ (see LeJan 1985). The problem dealt with in this work involves second order behavior of the flow. The evolving curvature of a submanifold moving under the flow would depend on the properties of the second order flow,

$$
(x, u, v) \rightarrow\left(\Phi_{t}(x), D \Phi_{t}(x) u, D^{2} \Phi_{t}(x)\left(D \Phi_{t}(x) u, D \Phi_{t}(x) u\right)+D \Phi_{t}(x) v\right) .
$$

In particular, our results rely on the properties given by (1.6), (1.7) and (1.8).

Initially, we will consider $M$ an embedded hypersurface in $\mathbb{R}^{n}$, later specializing to the case $n=3$. Take $\Pi_{t}: T_{x_{t}} \mathbb{R}^{n} \rightarrow T_{x_{t}} M_{t}$ to be the orthogonal projection and $\nabla$ to be the canonical connection on Euclidean space. Then $S_{t}$, the second fundamental form of $M_{t}$ at $x_{t}$, is defined for $u, v \in T_{x_{t}} M_{t}$ by $S_{t}(u, v)=\left(I-\Pi_{t}\right) \nabla_{u} v$ where $v$ is extended in an arbitrary but smooth manner in a neighborhood of $x_{t}$. Now $S_{t}$ induces a linear map from the exterior algebra $\Lambda^{k}\left(T_{x_{t}} M_{t}\right) \rightarrow \Lambda^{k}\left(T_{x_{t}} M_{t}\right)$ 
for $k=1,2, \ldots, n-1$. This arises as follows. First observe that since we are in Euclidean space we can identify vectors and forms. Next take $\nu_{t} \in T_{x_{t}}^{\perp} M_{t}$ to be a unit vector (there are two choices and we may select the process $\nu_{t}$ to be continuous in $t$.) Now in the case $k=1, u \rightarrow\left\langle S_{t}(u, \cdot), \nu_{t}\right\rangle$ is a map from $\Lambda^{1}\left(T_{x_{t}} M_{t}\right)$ to $\Lambda^{1}\left(T_{x_{t}} M_{t}\right)$. When $k>1$ we need to introduce the index set

$$
I_{k}=\left\{\vec{m} \in\{1, \ldots, n-1\}^{k}: m_{1}<m_{2}<\cdots<m_{k}\right\}
$$

and for $\sigma \in S_{k}$, the set of permutations on $k$ letters, define $(-1)^{\sigma}$ to be the sign of $\sigma$. Then for $u_{1}, \ldots, u_{k}, v_{1}, \ldots, v_{k} \in T_{x_{t}} M_{t}$, set

$$
S^{(k)}\left(u_{1} \wedge \cdots \wedge u_{k}, v_{1} \wedge \cdots \wedge v_{k}\right)=\sum_{\sigma \in S_{k}}(-1)^{\sigma} \prod_{j=1}^{k}\left\langle S_{t}\left(u_{\sigma(j)}, v_{j}\right), \nu_{t}\right\rangle
$$

This gives rise to the linear map

$$
u_{1} \wedge \cdots \wedge u_{k} \rightarrow S^{(k)}\left(u_{1} \wedge \cdots \wedge u_{k}, \cdot\right)
$$

from $\Lambda^{k}\left(T_{x_{t}} M_{t}\right) \rightarrow \Lambda^{k}\left(T_{x_{t}} M_{t}\right)$

The object of our study will be the trace of $S^{(k)}, \operatorname{Tr} S^{(k)}$, for $k=1,2, \ldots, n-1$. This is obtained via contraction on indices (see Bishop and Goldberg (1980)). Take $\left\{u_{1}, \ldots, u_{n-1}\right\}$ to be any basis for $T_{x_{t}} M_{t}$. For $k \in\{1, \ldots, n-1\}, \vec{\ell} \in I_{k}$, set $|\vec{\ell}|=\ell_{1}+\cdots+\ell_{k}$,

$$
\begin{aligned}
\alpha_{\vec{\ell}} & =u_{\ell_{1}} \wedge u_{\ell_{2}} \wedge \cdots \wedge u_{\ell_{k}} \\
\alpha^{\vec{\ell}} & =(-1)^{|\vec{\ell}|+k} u_{1} \wedge \cdots \wedge \hat{u}_{\ell_{1}} \wedge \cdots \wedge \hat{u}_{\ell_{k}} \wedge \cdots \wedge u_{n-1}
\end{aligned}
$$

( $\wedge$ indicates the indicated vector is omitted from the wedge product) $\alpha=u_{1} \wedge \cdots \wedge u_{n-1}$.

Define an inner product

$$
\left\langle\alpha_{\vec{\ell}}, \alpha_{\vec{m}}\right\rangle=\operatorname{det}\left\langle u_{\ell_{i}}, u_{m j}\right\rangle
$$

Then we may express $\operatorname{Tr} S^{(k)}$ as

$$
\operatorname{Tr} S^{(k)}=S^{(k)}\left(\alpha_{\ell}, \alpha_{\vec{m}}\right)\left\langle\alpha^{\vec{\ell}}, \alpha^{\vec{m}}\right\rangle \cdot\|\alpha\|^{-2}
$$

where the sum (Einstein's convention) is over $\vec{\ell}, \vec{m} \in I_{k}$. Now this is invariant under change of basis so we may select $\left\{u_{1}, \ldots, u_{n-1}\right\}$ to be the unit principal directions of curvature (see Spivak (1975).) Namely, the eigenvectors of $S^{(1)}: \Lambda^{1}\left(T_{x_{t}} M_{t}\right) \rightarrow$ 
$\Lambda^{1}\left(T_{x_{t}} M_{t}\right)$. Let $\lambda_{1}, \ldots, \lambda_{n-1}$ be the corresponding eigenvalues, otherwise known as the principal curvatures. Now the $u_{i}$ are orthonormal so

$$
\left\langle\alpha^{\vec{\ell}}, \alpha^{\vec{m}}\right\rangle\|\alpha\|^{-2}=\delta^{\vec{\ell}, \vec{m}}
$$

and as $\left\langle S_{t}\left(u_{j}, v\right), \nu_{t}\right\rangle=\lambda_{j}\left\langle u_{j}, v\right\rangle$

$$
\sum_{\sigma \in S_{k}}(-1)^{\sigma} \prod_{j=1}^{k}\left\langle S_{t}\left(u_{\ell_{\sigma(j)}}, u_{\ell_{j}}\right), \nu_{t}\right\rangle=\lambda_{\ell_{1}} \ldots \lambda_{\ell_{k}} \equiv \lambda_{\ell_{\ell}}
$$

Thus

$$
\operatorname{Tr} S^{(k)}=\sum_{\vec{\ell} \in I_{k}} \lambda_{\vec{\ell}} \equiv P_{k}\left(\lambda_{1}, \ldots, \lambda_{n-1}\right) .
$$

$P_{k}$ is the elementary symmetric polynomial of degree $k$.

The process $\xi_{t}=\left(x_{t}, \Pi_{t}, \nu_{t}, P_{1}\left(\lambda_{1}, \ldots, \lambda_{n-1}\right), \cdots P_{n-1}\left(\lambda_{1}, \ldots, \lambda_{n-1}\right)\right)$ will, in our case be a diffusion. By the translation invariance part of isotropy, it is clear that $x_{t}$ is not needed, i.e., $\left(\Pi_{t}, \nu_{t}, P_{1}\left(\lambda_{1}, \ldots, \lambda_{n-1}\right), \cdots, P_{n-1}\left(\lambda_{1}, \ldots, \lambda_{n-1}\right)\right)$ has the Markov property. By the invariance of the law of the flow under unitary actions, it follows that $\Pi_{t}$ and $\nu_{t}$ are also superfluous. Thus, for isotropic flows the vector $\left(P_{1}\left(\lambda_{1}, \ldots, \lambda_{n-1}\right), \ldots, P_{n-1}\left(\lambda_{1}, \ldots, \lambda_{n-1}\right)\right)$ is a diffusion. The point of the present article is to describe this diffusion explicitly in the measure-preserving isotropic case. We can derive consequences about the behavior of this diffusion from the explicit description. These properties suggest what sort of behavior one might have with more general flows. What we expect for more general flows is that the process of curvatures for a $k$-dimensional submanifold evolving under a general flow will be recurrent (though not necessarily a diffusion) if $\gamma_{k}>0$. That is, generically the $k$ dimensional tangent space $T_{x_{t}} M_{t}$ will 'see' only stretching directions and this will tend to smooth out the curvatures. This condition may even be too strong.

\section{$\S 2$ Preliminary Results.}

Recall the correlations of $d W$ given by (1.6). The flow $\Phi_{t}: \mathbb{R}^{n} \rightarrow \mathbb{R}^{n}$ is a $C^{\infty}$ diffeomorphism whose first derivative satisfies the Stratonovich stochastic differential equation, where $x \in M$,

$$
d D \Phi_{t}(x)=\partial W\left(x_{t}\right) D \Phi_{t}(x)
$$

Given $u \in T_{x} M, D \Phi_{t}(x) u=u(t) \in T_{x_{t}} M_{t}$ satisfies

$$
d u=\partial W u
$$

where we have suppressed the variable $\left(x_{t}\right)$ in $\partial W$. In fact, due to the choice of $C$ in $(1.1)$

$$
d u=d W u
$$


that is, the Itô corrections vanish. Furthermore, if we select a basis $\left\{u_{1}, \ldots, u_{n-1}\right\}$ for $T_{x} M$, then $\left\{u_{1}(t), \ldots, u_{n-1}(t)\right\}$, where $u_{j}(t)=D \Phi_{t}(x) u_{j}$, forms a basis for $T_{x_{t}} M_{t}$. We will write $u_{j}$ for $u_{j}(t)$ for simplicity of notation.

We now proceed to derive a Stratonovich equation for the second fundamental form $S_{t}$ of $M_{t}$ at $x_{t}$. Given vectors $u, v \in T_{x} M$ move them via the flow, $u(t)=$ $D \Phi_{t}(x) u, v(t)=D \Phi_{t}(x) v$. Extend $v$ to a smooth vector field $V$ in a neighborhood of $x$. Set $V_{t}(y)=D \Phi_{t}\left(\Phi_{t}^{-1}(y)\right) V\left(\Phi_{t}^{-1}(y)\right)$ so that $V_{t}$ is a vector field near $x_{t}$ such that $V_{t}\left(x_{t}\right)=v(t)$. Then if $Z_{t} \equiv \nabla_{u(t)} V_{t}\left(x_{t}\right)$ we claim

$$
d Z(u, v)=\partial W Z(u, v)+\partial B(u, v) .
$$

For proof of (2.4), take $\gamma$ to be a curve on $M$ with $\gamma(0)=x, \gamma^{\prime}(0)=u$ and define $\gamma_{t}=\Phi_{t}(\gamma)$ so that $\gamma_{t}^{\prime}(0)=u(t)=D \Phi_{t} u$. Then,

$$
\begin{aligned}
\nabla_{u_{t}} V_{t} & =\lim _{s \rightarrow 0} s^{-1}\left(V_{t}\left(\gamma_{t}(s)\right)-V_{t}\left(\gamma_{t}(0)\right)\right. \\
& =\lim _{s \rightarrow 0} s^{-1}\left(D \Phi_{t}(\gamma(s)) V(\gamma(s))-D \Phi_{t}(x) v(x)\right) \\
& =\lim _{s \rightarrow 0} s^{-1}\left[\left(D \Phi_{t}(\gamma(s))-D \Phi_{t}(x)\right) V(\gamma(s))\right. \\
& \left.+D \Phi_{t}(x)(V(\gamma(s))-v(x))\right] \\
& =D^{2} \Phi_{t}(x)(u, v)+D \Phi_{t}(x) \nabla_{u} v
\end{aligned}
$$

Since

$$
\begin{aligned}
\Phi_{t}(x) & =x+\int_{0}^{t} \partial U\left(\Phi_{s}(x)\right) \\
D \Phi_{t}(x) & =I+\int_{0}^{t} \partial W\left(\Phi_{s}(x)\right) D \Phi_{s}(x) \\
D^{2} \Phi_{t}(x)(\cdot, \cdot) & =\int_{0}^{t} \partial B\left(\Phi_{s}(x)\right)\left(D \Phi_{s}(x) \cdot D \Phi_{s}(x) \cdot\right) \\
& +\int_{0}^{t} \partial W\left(\Phi_{s}(x)\right) D^{2} \Phi_{s}(x)(\cdot, \cdot)
\end{aligned}
$$

(2.4) follows. A word about notation: $\partial W_{t} Z$ is an abbreviation for $\partial W_{j}^{i}\left(\Phi_{t}(x)\right)\left(Z_{t}^{j}\right)$ and $\partial B(u, v)$ is short for $\left(\partial B_{j k}^{i}\left(\Phi_{t}(x)\right) u_{j}(t) v_{k}(t)\right.$.

Next we need an equation for $\Pi_{t}: T_{x_{t}} \mathbb{R}^{n} \rightarrow T_{x_{t}} M_{t}$. For any $\xi \in T_{x_{t}} M_{t}$, let $\left\{u_{1}, \ldots, u_{n-1}\right\}$ be the moving frame mentioned above, set $\alpha=u_{1} \wedge \cdots \wedge u_{n-1}$ and defining

$$
\alpha^{i}=(-1)^{i+1} u_{1} \wedge \cdots \wedge \hat{u}_{i} \wedge \cdots \wedge u_{n-1}
$$

we have

$$
\Pi_{t} \xi=\sum_{i=1}^{n-1}\left\langle\xi \wedge \alpha^{i}, \alpha\right\rangle\|\alpha\|^{-2} u_{i} .
$$

The next two lemmas are elementary but crucial. 
Lemma 2.1. If $\xi_{t}$ is a $T_{x_{t}} M_{t}$-valued continuous semimartingale, then

$$
\left(I-\Pi_{t}\right) \cdot \partial W \xi_{t}=\partial \Pi_{t} \xi_{t}
$$

Proof. Suppose $\Pi_{t} \xi_{t}=\xi_{t}$ is as in the statement of the lemma with $\xi_{t}=x_{t}^{i} u_{i}$. Then

$$
\begin{aligned}
\left(I-\Pi_{t}\right) \partial W \xi_{t} & -\partial \Pi_{t} \xi_{t}=\partial W \xi_{t}-\Pi_{t} \partial W \xi_{t} \\
& +2 \frac{\left\langle\partial \alpha_{t}, \alpha_{t}\right\rangle}{\left\|\alpha_{t}\right\|^{2}} \xi_{t}-\partial W \xi_{t} \\
& -\frac{\left\langle\xi_{t} \wedge \alpha_{t}^{i}, \partial \alpha_{t}\right\rangle}{\left\|\alpha_{t}\right\|^{2}} u_{i}-\frac{\left\langle\xi_{t} \wedge \partial \alpha_{t}^{i}, \alpha_{t}\right\rangle}{\left\|\alpha_{t}\right\|^{2}} u_{i} \\
& =-\Pi_{t} \partial W \xi_{t}+2 \frac{\left\langle\partial \alpha_{t}, \alpha_{t}\right\rangle}{\left\|\alpha_{t}\right\|^{2}} \xi_{t} \\
& -x_{t}^{i} \frac{\left\langle\alpha_{t}, \partial \alpha_{t}\right\rangle}{\left\|\alpha_{t}\right\|^{2}} u_{i}-x_{t}^{i} \frac{\left\langle u_{i} \wedge \partial \alpha_{i}, \alpha_{t}\right\rangle}{\left\|\alpha_{t}\right\|^{2}} u_{i}
\end{aligned}
$$

Since

$$
d \alpha_{t}=-u_{i} \wedge \partial \alpha^{i}+\partial W u_{i} \wedge \alpha^{i}
$$

the last term becomes

$$
\begin{aligned}
& -x_{t}^{i} \frac{\left\langle u_{i} \wedge \partial \alpha^{i}, \alpha\right\rangle}{\left\|\alpha_{t}\right\|^{2}} u_{i} \\
& =-\frac{\left\langle\partial \alpha_{t}, \alpha_{t}\right\rangle}{\left\|\alpha_{t}\right\|^{2}} \xi_{t}+\frac{\left\langle\partial W u_{i} \wedge \alpha_{t}^{i}, \alpha_{t}\right\rangle}{\left\|\alpha_{t}\right\|^{2}} x_{t}^{i} u_{i} \\
& =-\frac{\left\langle\partial \alpha_{t}, \alpha_{t}\right\rangle}{\left\|\alpha_{t}\right\|^{2}} \xi_{t}+\Pi_{t} \partial W \xi_{t}
\end{aligned}
$$

From this we get the conclusion of the lemma.

Lemma 2.2. If $\eta_{t} \in T_{x_{t}} M_{t}^{\perp}$ is a continuous semimartingale, then

$$
\partial \Pi_{t} \eta_{t}=\Pi_{t} \partial \tilde{W} \eta_{t}
$$

where $\tilde{W}$ is the transpose of $W$.

Proof. Recalling that

$$
\begin{aligned}
\partial \Pi_{t}(v) & =-2 \frac{\left\langle\partial \alpha_{t}, \alpha_{t}\right\rangle}{\left\|\alpha_{t}\right\|^{2}} \Pi_{t} v+\partial W \Pi_{t} v \\
& +\frac{\left\langle v \wedge \alpha_{t}^{i}, \partial \alpha_{t}\right\rangle}{\left\|\alpha_{t}\right\|^{2}} u_{i}+\frac{\left\langle v \wedge \partial \alpha_{t}^{i}, \alpha_{t}\right\rangle}{\left\|\alpha_{t}\right\|^{2}} u_{i}
\end{aligned}
$$


we see that since $\Pi_{t} \eta_{t}=0$,

$$
\partial \Pi\left(\eta_{t}\right)=\frac{\left\langle\eta_{t} \wedge \alpha_{t}^{i}, \partial \alpha_{t}\right\rangle}{\left\|\alpha_{t}\right\|^{2}} u_{i}
$$

But

$$
\partial \alpha_{t}=\partial W u_{k} \wedge \alpha_{t}^{k}
$$

so

$$
\begin{aligned}
\partial \Pi_{t}\left(\eta_{t}\right) & =\frac{\left\langle\eta_{t} \wedge \alpha_{t}^{i}, \partial W u_{k} \wedge \alpha_{t}^{k}\right\rangle}{\left\|\alpha_{t}\right\|^{2}} u_{i} \\
& =\frac{\left\langle\partial \tilde{W} \eta_{t}, u_{k}(t)\right\rangle\left\langle\alpha_{t}^{k}(t), \alpha_{t}^{k}(t)\right\rangle u_{i}(t)}{\|\alpha(t)\|^{2}} \\
& =\frac{\left\langle\partial \tilde{W} \eta_{t} \wedge \alpha_{t}^{i}, \alpha_{t}\right\rangle}{\left\|\alpha_{t}\right\|^{2}} u_{t}^{i} \\
& =\Pi_{t} \partial \tilde{W} \eta_{t}
\end{aligned}
$$

and the lemma is proved.

Using $S=(I-\Pi) Z$ together with Lemmas 2.1 and 2.2 we obtain the following.

Proposition 2.3. For $u, v \in T_{x} M, u(t)=D \Phi_{t}(x) u, v(t)=D \Phi_{t}(x) v$,

$$
d S(u, v)=(I-\Pi) d B(u, v)+\partial W S(u, v)-\Pi(\partial W+\partial \tilde{W}) S(u, v)
$$

where $\tilde{W}$ denotes the transpose of $W$.

Proof. We shall abbreviate by suppressing the dependence on $u$ and $v$. So,

$$
\begin{aligned}
d S & =(I-\Pi) \partial Z-\partial \Pi Z \\
& =(I-\Pi) d B+(I-\Pi) \partial W Z-\partial \Pi Z, \text { by }(2.4), \\
& =(I-\Pi) d B+(I-\Pi) \partial W S \\
& +(I-\Pi) \partial W R-\partial \Pi R-\partial \Pi S \quad, \Pi Z \equiv R \\
& =(I-\Pi) d B+(I-\Pi) \partial W S-\partial \Pi S, \quad \text { by Lemma } 2.1 \\
& =(I-\Pi) d B+\partial W S-\Pi(\partial W+\partial \tilde{W}) S, \text { by Lemma } 2.2 .
\end{aligned}
$$

The equation (2.7) can be simplified. Set

$$
\begin{aligned}
& d P=(I-\Pi) d W \\
& d Q=\Pi d \tilde{W}
\end{aligned}
$$

and define $\lambda$ and $\mu$ so that

$$
\begin{array}{r}
(I-\Pi) \partial W=d P+d \lambda \\
\Pi \partial \tilde{W}=d Q+d \mu .
\end{array}
$$


Lemma 2.4.

$$
\begin{gathered}
d \Pi=d P \Pi+d \lambda \Pi+d Q(I-\Pi)+d \mu(I-\Pi) \\
d S(u, v)=(I-\Pi) d B(u, v)+(d P-d Q) S(u, v)+\frac{1}{2}(d P-d Q)^{2} S(u, v) \\
+d(\lambda-\mu) S(u, v)
\end{gathered}
$$

Proof. Let $\zeta_{t}$ be an $\mathbb{R}^{n}$-valued semimartingale. Then

$$
d \Pi=(I-\Pi) \partial W \Pi+\Pi \partial \tilde{W}(I-\Pi)
$$

holds since

$$
\begin{aligned}
\partial \Pi_{t} \zeta_{t} & =\partial \Pi_{t}\left(\Pi_{t} \zeta_{t}\right)+\partial \Pi_{t}\left(\left(I-\Pi_{t}\right) \zeta_{t}\right) \\
& =\left(I-\Pi_{t}\right) \partial W_{t} \Pi_{t} \zeta_{t}+\Pi_{t} \partial \tilde{W}_{t}\left(I-\Pi_{t}\right) \zeta_{t},
\end{aligned}
$$

by Lemmas 2.1 and 2.2. Thus, we have the following quadratic variations,

$$
\begin{aligned}
d \lambda & =\frac{1}{2}(-(I-\Pi) d W \Pi d W-\Pi d \tilde{W}(I-\Pi) d W) \\
& =\frac{1}{2}(-d P \Pi d W-d Q(I-\Pi) d W) \\
d \mu & =\frac{1}{2}((I-\Pi) d W \Pi d \tilde{W}+\Pi d \tilde{W}(I-\Pi) d \tilde{W}) \\
& =\frac{1}{2}(d P \Pi d \tilde{W}+d Q(I-\Pi) d \tilde{W}) .
\end{aligned}
$$

Consequently, performing Itô corrections on (2.12), we get

$$
d \Pi=d P \Pi+d \lambda \Pi+d Q(I-\Pi)+d \mu(I-\Pi)
$$

which proves (2.10). In order to derive (2.11), rewrite (2.7) as

$$
d S(u, v)=(I-\Pi) d B(u, v)+(I-\Pi) \partial W S(u, v)-\Pi \partial \tilde{W} S(u, v)
$$

Then (2.11) follows from the definitions of $d P, d Q, d \lambda$ and $d \mu$ in (2.9).

Lemma 2.5. If $\zeta_{t}$ is a $T_{x_{t}} M_{t}^{\perp}$-valued semi-martingale, then

$$
d \lambda \zeta=\frac{(n-1) \mu_{2}}{2 n(n+2)} \zeta d t
$$




$$
\begin{gathered}
d \mu \zeta=\frac{(n-1)(n+1) \mu_{2}}{2 n(n+2)} \zeta d t \\
\frac{1}{2}(d P-d Q)^{2} \zeta=-\frac{(n-1) n \mu_{2}}{2 n(n+2)} \zeta d t
\end{gathered}
$$

Proof. Select an orthonormal basis $\left\{e_{1}, \ldots, e_{n}\right\}$ for $T_{x_{t}} M_{t}$ such that $e_{n} \in T_{x_{t}} M_{t}^{\perp}$. Then,

$$
\begin{aligned}
2 d \lambda \zeta & =-d \Pi d W \zeta-d P \Pi d W \zeta-d Q(I-\Pi) d W \zeta \\
& =-(I-\Pi) d W \Pi d W \zeta-\Pi d \tilde{W}(I-\Pi) d W \zeta \\
& =-\left(\sum_{i=1}^{n-1} C_{n i}^{i n}\right) \zeta d t, \text { since }\left\langle d W_{i}^{n}, d W_{n}^{n}\right\rangle=0,1 \leq i \leq n-1, \\
& =\frac{(n-1) \mu_{2}}{n(n+2)} \zeta d t, \text { by }(1.6) \\
2 d \mu \zeta & =d P \Pi d \tilde{W} \zeta+d Q(I-\Pi) d \tilde{W} \zeta \\
& =\left(\sum_{i=1}^{n-1} C_{n n}^{i i}\right) \zeta d t \\
& =\frac{(n-1)(n+1) \mu_{2}}{n(n+2)} \zeta d t, \text { by }(1.6) .
\end{aligned}
$$

Thus,

$$
\begin{aligned}
\frac{1}{2}(d P-d Q)^{2} \zeta & =\frac{1}{2}(d P-d Q)((I-\Pi) d W \zeta-\Pi d \tilde{W} \zeta) \\
& =\frac{1}{2}(d P-d Q)\left(d W_{n}^{n} \zeta-\sum_{i=1}^{n-1} d \tilde{W}_{i}^{n} e_{i}\left\langle\zeta, e_{n}\right\rangle\right) \\
& =\frac{1}{2}\left(d W_{n}^{n} d W_{n}^{n} \zeta-\sum_{i=1}^{n-1} d W_{n}^{i} d \tilde{W}_{i}^{n} \zeta\right) \\
& =\frac{1}{2}\left(C_{n n}^{n n} \zeta-\left(\sum_{i=1}^{n-1} C_{n n}^{i i}\right) \zeta\right) d t \\
& =\frac{\mu_{2}}{2 n(n+2)}((n-1)-(n-1)(n+1)) \zeta d t, \text { by }(1.6), \\
& =-\frac{(n-1) \mu_{2}}{2(n+2)} \zeta d t .
\end{aligned}
$$

$\square$ Recall that we have selected an initial basis for $T_{x} M$ with $u_{n} \in T_{x} M^{\perp}$ and $u_{i}(t)=D \Phi_{t}(x) u_{i}$ gives a basis for $T_{x_{t}} M_{t}$ when $i$ runs through $\{1, \ldots, n-1\}$. 
Theorem 2.6. If $\nu(t)$ denotes the unit vector $\nu=\frac{(I-\Pi) u_{n}}{\left\|(I-\Pi) u_{n}\right\|}$ perpendicular to $M_{t}$ at $x_{t}$, then

$$
d \nu=-d Q \nu-\frac{\left(n^{2}-1\right) \mu_{2}}{2 n(n+2)} \nu d t
$$

Proof. Set $v_{n}=(I-\Pi) u_{n}$. Then by Lemmas 2.2 and 2.3,

$$
\begin{aligned}
d v_{n} & =-\Pi \partial \tilde{W} v_{n}+(I-\Pi) \partial W v_{n} \\
& =(d P-d Q) v_{n}+\frac{1}{2}(d P-d Q)^{2} v_{n}+(d \lambda-d \mu) v_{n} \\
& =(d P-d Q) v_{n}-\frac{(n-1) \mu_{2}}{(n+2)} v_{n} d t, \text { by Lemma } 2.5
\end{aligned}
$$

Then, applying

$$
\begin{aligned}
d\left\|v_{n}\right\|^{2} & =2\left\langle v_{n}, d v_{n}\right\rangle+\left\langle(d P-d Q) v_{n}\right\rangle \\
& =2\left\langle v_{n},(d P-d Q) v_{n}\right\rangle-\frac{2(n-1) \mu_{2}}{(n+2)}\left\|v_{n}\right\|^{2} d t+\left\langle(d P-d Q) v_{n}\right\rangle \\
& =2\left\|v_{n}\right\|^{2} d W_{n}^{n}-\frac{2(n-1) \mu_{2}}{(n+2)}\left\|v_{n}\right\|^{2} d t+\frac{(n-1) \mu_{2}}{n}\left\|v_{n}\right\|^{2} d t \\
& =2\left\|v_{n}\right\|^{2} d W_{n}^{n}-\frac{(n-1)(n-2) \mu_{2}}{n(n+2)}\left\|v_{n}\right\|^{2} d t
\end{aligned}
$$

and so

$$
d\left\|v_{n}\right\|^{-1}=-\left\|v_{n}\right\|^{-1} d W_{n}^{n}+\frac{(n-1)(n+1) \mu_{2}}{2 n(n+2)}\left\|v_{n}\right\|^{-1} d t .
$$

Consequently, as $\nu=\frac{v_{n}}{\left\|v_{n}\right\|}$,

$$
\begin{aligned}
d \nu= & (d P-d Q) \nu-\frac{(n-1) \mu_{2}}{(n+2)} \nu d t-(I-\Pi) d W \nu+\frac{(n-1)(n+1) \mu_{2}}{2 n(n+2)} \nu d t \\
& \quad-\left\langle(d P-d Q) \nu, d W_{n}^{n}\right\rangle \\
= & -d Q \nu-\frac{(n-1)^{2} \mu_{2}}{2 n(n+2)} \nu d t-\frac{(n-1) \mu_{2}}{n(n+2)} \nu d t \\
= & d Q \nu-\frac{(n+1)(n-1) \mu_{2}}{2 n(n+2)} \nu d t, \text { as desired. }
\end{aligned}
$$

We can now derive the equation for $\langle S(u, v), \nu\rangle$. 
Theorem 2.7. Suppose $u(t)=D \Phi_{t} u, v(t)=D \Phi_{t} v$ and $\nu$ is as in Theorem 2.6. Then

$$
d\langle S(u, v), \nu\rangle=\langle d B(u, v), \nu\rangle+\langle S(u, v), \nu\rangle\langle d W \nu, \nu\rangle-\frac{(n-1)^{2} \mu_{2}}{2 n(n+2)}\langle S(u, v), \nu\rangle d t .
$$

Moreover, if $u_{i}(t)=D \Phi_{t} u_{i}$, then

$$
\begin{aligned}
\left\langle d\left\langle S\left(u_{i}, u_{j}\right), \nu\right\rangle, d\left\langle S\left(u_{k}, u_{\ell}\right), \nu\right\rangle\right\rangle & =C_{1122}^{33}\left[\left\langle u_{i}, u_{j}\right\rangle\left\langle u_{k}, u_{\ell}\right\rangle\right. \\
& \left.+\left\langle u_{i}, u_{k}\right\rangle\left\langle u_{j}, u_{\ell}\right\rangle+\left\langle u_{i}, u_{\ell}\right\rangle\left\langle u_{j}, u_{k}\right\rangle\right] d t \\
& +\frac{\mu_{2}(n-1)}{n(n+2)}\left\langle S\left(u_{i}, u_{j}\right), \nu\right\rangle\left\langle S\left(u_{k}, u_{\ell}\right\rangle, \nu\right\rangle d t .
\end{aligned}
$$

Proof. From Lemmas 2.4 and 2.5 we have

$$
d S(u, v)=(I-\Pi) d B(u, v)+(d P-d Q) S(u, v)-\frac{(n-1) \mu_{2}}{(n+2)} S(u, v) d t .
$$

Also

$$
\begin{aligned}
-\langle(d P-d Q) S(u, v), d Q \nu\rangle & =\langle d Q S(u, v), d Q \nu\rangle \\
& =\langle S(u, v), \nu\rangle \sum_{i=1}^{n-1} C_{n n}^{i i} d t \\
& =\frac{\left(n^{2}-1\right) \mu_{2}}{n(n+2)}\langle S(u, v), \nu\rangle d t
\end{aligned}
$$

So,

$$
\begin{aligned}
d\langle S(u, v), \nu\rangle & =\langle d S(u, v), \nu\rangle+\langle S(u, v), d \nu\rangle+\langle d S(u, v), d \nu\rangle \\
& =\langle d B(u, v), \nu\rangle+\langle S(u, v), \nu\rangle d W_{n}^{n}-\frac{(n-1) \mu_{2}}{(n+2)}\langle S(u, v), \nu\rangle d t \\
& -\frac{\left(n^{2}-1\right) \mu_{2}}{2 n(n+2)}\langle S(u, v), \nu\rangle d t-\langle(d P-d Q) S(u, v), d Q \nu\rangle \\
& =\langle d B(u, v), \nu\rangle+\langle S(u, v), \nu\rangle d W_{n}^{n}-\frac{(n-1)(n-1)^{2} \mu_{2}}{2 n(n+2)}\langle S(u, v), \nu\rangle d t
\end{aligned}
$$

For the quadratic variation term, since $B$ and $W$ are independent and $C_{111}^{n n}=$ $3 C_{1122}^{n n}$,

$$
\begin{aligned}
\left\langle d S\left(u_{i}, u_{j}\right), \nu\right\rangle\left\langle d S\left(u_{k}, u_{\ell}\right), \nu\right\rangle & =\left\langle\left\langle d B\left(u_{i}, u_{j}\right), \nu\right\rangle\left\langle d B\left(u_{k}, u_{\ell}\right), \nu\right\rangle\right\rangle \\
& +\left\langle S\left(u_{i}, u_{j}\right), \nu\right\rangle\left\langle S\left(u_{k}, u_{\ell}\right), \nu\right\rangle C_{n n}^{n n} d t \\
& =C_{1122}^{n n}\left[\left\langle u_{i}, u_{j}\right\rangle\left\langle u_{k}, u_{\ell}\right\rangle+\left\langle u_{i}, u_{k}\right\rangle\left\langle u_{j}, u_{\ell}\right\rangle\right. \\
& \left.+\left\langle u_{i}, u_{\ell}\right\rangle\left\langle u_{j}, u_{k}\right\rangle\right] d t \\
& +\frac{(n-1) \mu_{2}}{n(n+2)}\left\langle S\left(u_{i}, u_{j}\right), \nu\right\rangle\left\langle S\left(u_{k}, u_{\ell}\right), \nu\right\rangle d t
\end{aligned}
$$


We now split the exposition. The case of a hypersurface in $\mathbb{R}^{3}$ will be developed below. The case of a hypersurface in $\mathbb{R}^{n}, n>3$, will be treated in an addendum. The latter involves lengthy computations together with combinatorial arguments, whereas the former is more readable.

Theorem 2.8. Let $u, v \in T_{x} M$ with $u$ and $v$ linearly independent and define $u(t)=D \Phi_{t} u, v(t)=D \Phi_{t} v$, (however in our computations we shall suppress the $t$ dependence). The process $\left(\operatorname{Tr} S^{(1)}, \operatorname{Tr} S^{(2)}\right)$ satisfies the equations

$$
\begin{aligned}
d \operatorname{Tr} S^{(1)}= & {\left[\langle d B(u, u), \nu\rangle\|v\|^{2}+\langle d B(v, v), \nu\rangle\|u\|^{2}-2\langle d B(u, v), \nu\rangle\langle u, v\rangle\right]\|\alpha\|^{-2} } \\
+ & \operatorname{Tr} S^{(1)}\left(d W_{3}^{3}-2\left(d W_{1}^{1}+d W_{2}^{2}\right)\right) \\
+ & 2[S(u, u)\langle d W v, v\rangle+S(v, v)\langle d W u, u\rangle-S(u, v)(\langle d W u, v\rangle+\langle u, d W v\rangle)]\|\alpha\|^{-2} \\
+ & \frac{4 \mu_{2}}{15} \operatorname{Tr} S^{(1)} d t \\
d \operatorname{Tr} S^{(2)}= & {[S(v, v)\langle d B(u, u), \nu\rangle+S(u, u)\langle d B(v, v), \nu\rangle-2 S(u, v)\langle d B(u, v), \nu\rangle]\|\alpha\|^{-2} } \\
+ & 2 \operatorname{Tr} S^{(2)}\left(d W_{3}^{3}-\left(d W_{1}^{1}+d W_{2}^{2}\right)\right) \\
+ & \frac{4 \mu_{2}}{15} \operatorname{Tr} S^{(2)} d t . \\
& \left\langle d \operatorname{Tr} S^{(1)}\right\rangle=\left[\frac{16 \mu_{4}}{35}+\frac{\mu_{2}}{15}\left[14\left(\operatorname{Tr} S^{(1)}\right)^{2}-24 \operatorname{Tr} S^{(2)}\right]\right] d t \\
& \left\langle d \operatorname{Tr} S^{(2)}\right\rangle=\left[\frac{2 \mu_{4}}{35}\left(3\left(\operatorname{Tr} S^{(1)}\right)^{2}-4 \operatorname{Tr} S^{(2)}\right)+\frac{32 \mu_{2}}{15}\left(\operatorname{Tr} S^{(2)}\right)^{2}\right] d t \\
\left\langle d \operatorname{Tr} S^{(1)},\right. & \left.d \operatorname{Tr} S^{(2)}\right\rangle=\left[\frac{8 \mu_{4}}{35} \operatorname{Tr} S^{(1)}+\frac{16 \mu_{2}}{15} \operatorname{Tr} S^{(1)} \operatorname{Tr} S^{(2)}\right] d t .
\end{aligned}
$$

Proof. Recall we have selected an orthomal basis $\left\{e_{1}, e_{2}, e_{3}\right\}$ for $T_{x_{t}} M_{t}$ such that $\nu=e_{3}$. Then with $\alpha=u \wedge v$, from LeJan (1986) we have

$$
\frac{d\|\alpha\|^{-2}}{\|\alpha\|^{-2}}=-2\left(d W_{1}^{1}+d W_{2}^{2}\right)-\frac{2 \mu_{2}}{15} d t
$$

Using (1.6), it follows that

$$
d\langle u, v\rangle=\langle d W u, v\rangle+\langle u, d W v\rangle+\frac{10 \mu_{2}}{15}\langle u, v\rangle d t
$$

Now by (1.9), the mean curvature is

$$
\operatorname{Tr} S^{(1)}=\left[S(u, u)\|v\|^{2}+S(v, v)\|u\|^{2}-2 S(u, v)\langle u, v\rangle\right]\|\alpha\|^{-2}
$$


so by Itô's formula,

$$
\begin{aligned}
d \operatorname{Tr} S^{(1)}= & {\left[\|v\|^{2} d S(u, u)+\|u\|^{2} d S(v, v)-2\langle u, v\rangle d S(u, v)\right.} \\
& +S(u, u) d\|v\|^{2}+S(v, v) d\|u\|^{2}-2 S(u, v) d\langle u, v\rangle \\
& +\left\langle d S(u, u), d\|v\|^{2}\right\rangle+\left\langle d S(v, v), d\|u\|^{2}\right\rangle-2\langle d S(u, v), d\langle(u, v)\rangle]\|\alpha\|^{-2} \\
& +\operatorname{Tr} S^{(1)} \frac{d\|\alpha\|^{-2}}{\|\alpha\|^{-2}}+\left\langle d\left(\operatorname{Tr} S^{(1)}\|\alpha\|^{2}\right), d\|\alpha\|^{-2}\right\rangle \\
= & {\left[\langle d B(u, u), \nu\rangle\|v\|^{2}+\langle d B(v, v), \nu\rangle\|u\|^{2}-2\langle d B(u, v), \nu\rangle\langle u, v\rangle\right]\|\alpha\|^{-2} } \\
& +\operatorname{Tr} S^{(1)} d W_{3}^{3}-\frac{2 \mu_{2}}{15} \operatorname{Tr} S^{(1)} d t \\
& +[S(u, u)\langle d W v, v\rangle+S(v, v)\langle d W u, u\rangle-S(u, v)(\langle d W u, v\rangle+\langle u, d W v\rangle)]\|\alpha\|^{-2} \\
+ & \frac{10 \mu_{2}}{15} \operatorname{Tr} S^{(1)} d t-\frac{2 \mu_{2}}{15} \operatorname{Tr} S^{(1)} d t \\
- & 2 \operatorname{Tr} S^{(1)}\left(d W_{1}^{1}+d W_{2}^{2}\right)-\frac{2 \mu_{2}}{15} \operatorname{Tr} S^{(1)} d t,
\end{aligned}
$$

using Theorem 2.7, (2.15), (2.16) and the fact that

$$
\begin{aligned}
\left\langle d\left(\operatorname{Tr} S^{(1)}\|\alpha\|^{2}\right), d\|\alpha\|^{-2}\right\rangle= & -2\|\alpha\|^{-2}\left[\left\langle\|\alpha\|^{2} \operatorname{Tr} S^{(1)} d W_{3}^{3}, d W_{1}^{1}+d W_{2}^{2}\right\rangle\right. \\
& +2\langle S(u, u)\langle d W v, v\rangle+S(v, v)\langle d W u, u\rangle \\
- & \left.\left.S(u, v)(\langle d W u, v\rangle+\langle u, d W v\rangle), d W_{1}^{1}+d W_{2}^{2}\right\rangle\right] \\
= & -2\left[\frac{-2 \mu_{2}}{15}+\frac{2 \mu_{2}}{15}\right] \operatorname{Tr} S^{(1)} d t \\
= & 0 .
\end{aligned}
$$

Thus,

$$
\begin{aligned}
d \operatorname{Tr} S^{(1)} & =\left[\langle d B(u, u), \nu\rangle\|v\|^{2}+\langle d B(v, v), \nu\rangle\|u\|^{2}-2\langle d B(u, v), \nu\rangle\langle u, v\rangle\right]\|\alpha\|^{-2} \\
& +\operatorname{Tr} S^{(1)}\left(d W_{3}^{3}-2\left(d W_{1}^{1}+d W_{2}^{2}\right)\right) \\
& +2[S(u, u)\langle d W v, v\rangle+S(v, v)\langle d W u, u\rangle-S(u, v)(\langle d W u, v\rangle \\
& +\langle u, d W v\rangle)]\|\alpha\|^{-2} \\
& +\frac{4 \mu_{2}}{15} \operatorname{Tr} S^{(1)} d t
\end{aligned}
$$

Consequently, the quadratic variation of $\operatorname{Tr} S^{(1)}$ is given by

$$
\begin{aligned}
\left\langle d \operatorname{Tr} S^{(1)}\right\rangle & =\left[\langle d B(u, u), \nu\rangle\|v\|^{2}+\langle d B(v, v), \nu\rangle\|u\|^{2}-2\langle d B(u, v), \nu\rangle\langle u, v\rangle\right]\|\alpha\|^{-4} \\
& +\left(\operatorname{Tr} S^{(1)}\right)^{2}\left\langle d W_{3}^{3}-2\left(d W_{1}^{1}+d W_{2}^{2}\right)\right\rangle \\
& +4[S(u, u)\langle d W v, v\rangle+S(v, v)\langle d W u, u\rangle-S(u, v)(\langle d W u, v\rangle+\langle u, d W v\rangle)]\|\alpha\|^{-4} \\
& +4 \operatorname{Tr} S^{(1)}\left\langle d W_{3}^{3}-2\left(d W_{1}^{1}+d W_{2}^{2}\right), S(u, u)\langle d W v, v\rangle+S(v, v)\langle d W u, u\rangle\right. \\
& -S(u, v)(\langle d W u, v\rangle+\langle u, d W v\rangle)\rangle\|\alpha\|^{-2}
\end{aligned}
$$

Setting

$$
q_{n}=\frac{(n+3) \mu_{4}}{n(n+2)(n+4)}=C_{1122}^{n n}
$$


and if $u_{i}, u_{j}, u_{k}, u_{\ell}$ are all orthogonal to $\nu$, then

$$
\begin{aligned}
\left\langle d B\left(u_{i}, u_{j}\right), \nu\right\rangle\left\langle d B\left(u_{k}, u_{\ell}\right), \nu\right\rangle & =q_{n}\left[\left\langle u_{i}, u_{j}\right\rangle\left\langle u_{k}, u_{\ell}\right\rangle+\left\langle u_{i}, u_{k}\right\rangle\left\langle u_{j}, u_{\ell}\right\rangle\right. \\
& \left.+\left\langle u_{i}, u_{\ell}\right\rangle\left\langle u_{j}, u_{k}\right\rangle\right] d t
\end{aligned}
$$

Then

$$
\begin{aligned}
& \left\langle\langle d B(u, u), \nu\rangle\|v\|^{2}+\langle d B(v, v), \nu\rangle\|u\|^{2}-2\langle d B(u, v), \nu\rangle\langle u, v\rangle\right\rangle\|\alpha\|^{-4} \\
& =q_{3}\left[3\|u\|^{4}\|v\|^{4}+3\|v\|^{4}\|u\|^{4}+4\left(2\langle u, v\rangle^{2}+\|u\|^{2}\|v\|^{2}\right)\langle u, v\rangle^{2}\right. \\
& \quad+2\left(\|u\|^{2}\|v\|^{2}+2\langle u, v\rangle^{2}\right)\|u\|^{2}\|v\|^{2}-4\left(3\|u\|^{2}\langle u, v\rangle\right)\|v\|^{2}\langle u, v\rangle \\
& \quad-4\left(3\|v\|^{2}\langle u, v\rangle\right)\|u\|^{2}\langle u, v \|]\|\alpha\|^{-4} d t \\
& =q_{3}\left[8\|u\|^{4}-16\|u\|^{2}\|v\|^{2}\langle u, v\rangle^{2}+8\langle u, v\rangle^{4}\right]\|\alpha\|^{-4} d t \\
& =8 q_{3} d t .
\end{aligned}
$$

Next, observe

$$
\begin{aligned}
\left\langle d W_{3}^{3}-2\left(d W_{1}^{1}+d W_{2}^{2}\right)\right\rangle & =\left\langle d W_{3}^{3}\right\rangle-4\left\langle W_{3}^{3}, d W_{1}^{1}+d W_{2}^{2}\right\rangle+4\left\langle d W_{1}^{1}+d W_{2}^{2}\right\rangle \\
& =\frac{18 \mu_{2}}{15} d t
\end{aligned}
$$

and

$$
\begin{aligned}
\langle\langle d W v, v\rangle\rangle & =\frac{2 \mu_{2}}{15}\|v\|^{4} d t \\
\langle\langle d W v, v\rangle\langle d W u, u\rangle\rangle & =\frac{\mu_{2}}{15}\left(3\langle u, v\rangle^{2}-\|u\|^{2}\|v\|^{2}\right) d t \\
\langle\langle d W u, v\rangle,\langle u, d W v\rangle\rangle & =\frac{\mu_{2}}{15}\left(3\langle u, v\rangle^{2}-\|v\|^{2}\right) d t \\
\langle\langle d W u, v\rangle,\langle d W u, v\rangle\rangle & =\frac{\mu_{2}}{15}\left(4\|u\|^{2}\|v\|^{2}-2\langle u, v\rangle^{2}\right) d t \\
\langle\langle d W u, v\rangle,\langle d W v, v\rangle\rangle & =\frac{2 \mu_{2}}{15}\langle u, v\rangle\|v\|^{2} d t \\
\langle\langle d W v, u\rangle,\langle d W u, u\rangle\rangle & =\frac{2 \mu_{2}}{15}\langle u, v\rangle\|u\|^{2} d t \\
\langle d W v, u\rangle\langle v, v\rangle & =\frac{2 \mu_{2}}{15}\langle u, v\rangle\|v\|^{2} d t
\end{aligned}
$$


Thus,

$$
\begin{aligned}
& {[S(u, u)\langle d W v, v\rangle+S(v, v)\langle d W u, u\rangle-S(u, v)(\langle d W u, v\rangle+\langle u, d W v\rangle)]} \\
& \begin{array}{l}
\mu_{2} \\
15
\end{array} 2 S(u, u)^{2}\|v\|^{4}+2 S(v, v)^{2}\|u\|^{4} \\
& \quad+2 S(u, v)^{2}\left(3\|u\|^{2}\|v\|^{2}+\langle u, v\rangle^{2}\right)+2 S(u, u) S(v, v)\left(3\langle u, v\rangle^{2}-\|u\|^{2}\|v\|^{2}\right) \\
& \quad-2 S(u, u) S(u, v)\left(1\langle u, v\rangle\|u\|^{2}+2\langle u, v\rangle\|u\|^{2}\right)\|v\|^{2} \\
& \left.\quad-2 S(v, v) S(u, v)\left(2\langle u, v\rangle\|u\|^{2}+2\langle u, v\rangle\|u\|^{2}\right)\|u\|^{2}\right] d t \\
& =\frac{\mu_{2}}{15}\left[2 \left\{S(u, u)^{2}\|v\|^{4}+S(v, v)^{2}\|u\|^{4}+2 S(u, u) S(v, v)\|u\|^{2}\|v\|^{2}\right.\right. \\
& \quad-4 S(u, u) S(u, v)\|u\|^{2}\|v\|^{2}\langle u, v\rangle \\
& \quad-4 S(v, v) S(u, v)\|u\|^{2}\|v\|^{2}\langle u, v\rangle \\
& \left.\quad 4 S(u, v)^{2}\langle u, v\rangle^{2}\right\} \\
& \left.\quad-6\left(S(u, u) S(v, v)-S(u, v)^{2}\right)\|\alpha\|^{2}\right] d t \\
& =\frac{\mu_{2}}{15}\left[2\left(\operatorname{Tr} S^{(1)}\right)^{2}-6 \operatorname{Tr} S^{(2)}\right]\|\alpha\|^{4} d t
\end{aligned}
$$

It's easy to verify that

$$
\begin{aligned}
\left\langle d W_{3}^{3},\langle d W v, v\rangle\right\rangle & =-\frac{\mu_{2}}{15}\|v\|^{2} d t \\
\left\langle d W_{3}^{3},\langle d W u, v\rangle\right\rangle & =\left\langle d W_{3}^{3},\langle u, d W v\rangle\right\rangle=-\frac{\mu_{2}}{15}\langle u, v\rangle d t \\
\left\langle d W_{1}^{1}+d W_{2}^{2},\langle d W u, v\rangle\right\rangle & =\frac{\mu_{2}}{15}\langle u, v\rangle d t
\end{aligned}
$$

so

$$
\begin{aligned}
& \left\langle d W_{3}^{3}, S(u, u)\langle d W v, v\rangle+S(v, v)\langle d W u, u\rangle-S(u, v)(\langle d W u, v\rangle+\langle u, d W v\rangle)\right\rangle \\
& =-\frac{\mu_{2}}{15}\left[S(u, u)\|v\|^{2}+S(v, v)\|u\|^{2}-2 S(u, v)\langle u, v\rangle\right] d t \\
& =-\frac{\mu_{2}}{15} \operatorname{Tr} S^{(1)}\|\alpha\|^{2} d t \\
& \left\langle d W_{1}^{1}+d W_{2}^{2}, S(u, u)\langle d W v, v\rangle+S(v, v)\langle d W u, u\rangle-S(u, v)(\langle d W u, v\rangle+\langle u, d W v\rangle)\right\rangle \\
& =\frac{\mu_{2}}{15}\left[S(u, u)\|v\|^{2}+S(v, v)\|u\|^{2}-2 S(u, v)\langle u, v\rangle\right] d t \\
& =\frac{\mu_{2}}{15} \operatorname{Tr} S^{(1)}\|\alpha\|^{2} d t .
\end{aligned}
$$

Therefore

$$
4 \operatorname{Tr} S^{(1)}\|\alpha\|^{-2}\left\langle d W_{3}^{3}-2\left(d W_{1}^{1}+d W_{2}^{2}\right), S(u, u)\langle d W v, v\rangle+S(v, v)\langle d W u, u\rangle\right.
$$

$$
\begin{aligned}
& -S(u, v)(\langle d W u, v\rangle+\langle u, d W v\rangle)\rangle \\
& =4\left(\operatorname{Tr} S^{(1)}\right)^{2}\left[-\frac{\mu_{2}}{15}-\frac{2 \mu_{2}}{15}\right] d t \\
& =-\frac{12 \mu_{2}}{15}\left(\operatorname{Tr} S^{(1)}\right)^{2} d t
\end{aligned}
$$


Combining (2.20), (2.21) and (2.22) we arrive at

$$
\left\langle d \operatorname{Tr} S^{(1)}\right\rangle=\frac{16 \mu_{4}}{35} d t+\frac{\mu_{2}}{15}\left[14\left(\operatorname{Tr} S^{(1)}\right)^{2}-24 \operatorname{Tr} S^{(2)}\right] d t .
$$

Turning now to the Gauss curvature, by (1.9) we have,

$$
\operatorname{Tr} S^{(2)}=\left[S(u, u) S(v, v)-S(u, v)^{2}\right]\|\alpha\|^{-2} .
$$

So, by Itô's formula,

$$
\begin{aligned}
d \operatorname{Tr} S^{(2)}= & (S(v, v) d S(u, u)+S(u, u) d S(v, v)-2 S(u, v) d S(u, v) \\
& +\langle d S(u, u), d S(v, v)\rangle-\langle d S(u, v), d S(u, v)\rangle]\|\alpha\|^{-2} \\
& +\left[S(u, u) S(v, v)-S(u, v)^{2}\right] d\|\alpha\|^{-2} \\
& +\left\langle S(v, v) d S(u, u)+S(u, u) d S(v, v)-2 S(u, v) d S(u, v), d\|\alpha\|^{-2}\right\rangle \\
= & {[S(v, v)\langle d B(u, u), \nu\rangle+S(u, u)\langle d B(v, v), \nu\rangle} \\
& -2 S(u, v)\langle d B(u, v), \nu\rangle]\|\alpha\|^{-2} \\
& +2 \operatorname{Tr} S^{(2)} d W_{3}^{3}-\frac{4 \mu_{2}}{15} \operatorname{Tr} S^{(2)} d t \\
& +[\langle\langle d B(u, u), \nu\rangle,\langle d B(v, v), \nu\rangle\rangle-\langle d B(u, v), \nu\rangle,\langle d B(u, v), \nu\rangle\rangle]\|\alpha\|^{-2} d t \\
& +\frac{2 \mu_{2}}{15} \operatorname{Tr} S^{(2)} d t \\
& -2 \operatorname{Tr} S^{(2)}\left(d W_{1}^{1}+d W_{2}^{2}\right)-\frac{2 \mu_{2}}{15} \operatorname{Tr} S^{(2)} d t \\
& \frac{8 \mu_{2}}{15} \operatorname{Tr} S^{(2)} d t .
\end{aligned}
$$

Thus,

$$
\begin{aligned}
d \operatorname{Tr} S^{(2)}= & {[S(v, v)\langle d B(u, u), \nu\rangle+S(u, u)\langle d B(v, v), \nu\rangle} \\
& -2 S(u, v)\langle d B(u, v), \nu\rangle]\|\alpha\|^{-2} \\
+ & 2 \operatorname{Tr} S^{(2)}\left(d W_{3}^{3}-\left(d W_{1}^{1}+d W_{2}^{2}\right)\right)+\frac{4 \mu_{2}}{15} \operatorname{Tr} S^{(2)} d t
\end{aligned}
$$

since $\langle\langle d B(u, u), \nu\rangle,\langle d B(v, v), \nu\rangle\rangle=\langle(d B(u, v), \nu\rangle,\langle d B(u, v), \nu\rangle\rangle$. 
Using (2.20), (2.26) and our remarks above on $W$ correlations, we get

$$
\begin{aligned}
\left\langle d \operatorname{Tr} S^{(2)}\right\rangle= & \langle S(v, v)\langle d B(u, u), \nu\rangle+S(u, u)\langle d B(v, v), \nu\rangle \\
& -2 S(u, v)\langle d B(u, v), \nu\rangle\rangle\|\alpha\|^{-4}+4\left(\operatorname{Tr} S^{(2)}\right)^{2}\left\langle d W^{3}-\left(d W_{1}^{1}+d W_{2}^{2}\right)\right\rangle \\
= & q_{3}\left[3 S(v, v)^{2}\|u\|^{4}+3 S(u, u)^{2}\|v\|^{4}+4 S(u, v)^{2}\left(\|u\|^{2}\|v\|^{2}+2\langle u, v\rangle^{2}\right)\right. \\
& +2 S(u, u) S(v, v)\left(\|u\|^{2}\|v\|^{2}+2\langle u, v\rangle^{2}\right) \\
& \left.-12 S(v, v) S(u, v)\|u\|^{2}\langle u, v\rangle-12 S(u, u) S(u, v)\|v\|^{2}\langle u, v\rangle\right]\|\alpha\|^{-2} d t \\
+ & \frac{32 \mu_{2}}{15}\left(\operatorname{Tr} S^{(2)}\right)^{2} d t \\
= & 3 q_{3}\left(\operatorname{Tr} S^{(1)}\right)^{2} d t-4 q_{3} \operatorname{Tr} S^{(2)} d t+\frac{32 \mu_{2}}{15}\left(\operatorname{Tr} S^{(2)}\right)^{2} d t \\
= & \frac{2 \mu_{4}}{35}\left(3\left(\operatorname{Tr} S^{(1)}\right)^{2}-4 \operatorname{Tr} S^{(2)}\right) d t+\frac{32 \mu_{2}}{15}\left(\operatorname{Tr} S^{(2)}\right)^{2} d t
\end{aligned}
$$

Finally we need

$$
\begin{aligned}
\left\langle d \operatorname{Tr} S^{(1)}, d \operatorname{Tr} S^{(2)}\right\rangle= & 2 \operatorname{Tr} S^{(1)} \operatorname{Tr} S^{(2)}\left\langle d W_{3}^{3}-2\left(d W_{1}^{1}+d W_{2}^{2}\right), d W_{3}^{3}\right. \\
& \left.-\left(d W_{1}^{1}+d W_{2}^{2}\right)\right\rangle+4 \operatorname{Tr} S^{(2)}\langle S(u, u)\langle d W v, v) \\
& +S(v, v)\langle W u, u\rangle-S(u, v)(\langle d W u, v\rangle \\
& \left.+\langle u, d W v\rangle, d W_{3}^{3}-\left(d W_{1}^{1}+d W_{2}^{2}\right)\right\rangle\|\alpha\|^{-2} \\
& +\|\alpha\|^{-4}\left\langle\langle d B(u, u), \nu\rangle\|v\|^{2}+\langle d B(v, v), \nu\rangle\|u\|^{2}\right. \\
& -2\langle d B(u, v), \nu\rangle\langle u, v\rangle, S(v, v)\langle d B(u, u), \nu\rangle \\
& +S(u, u)\langle d B(v, v), \nu\rangle-2 S(u, v)\langle d B(u, v), \nu\rangle\rangle \\
= & \frac{24 \mu_{2}}{15} \operatorname{Tr} S^{(1)} \operatorname{Tr} S^{(2)} d t-\frac{8 \mu_{2}}{15} \operatorname{Tr} S^{(1)} \operatorname{Tr} S^{(2)} d t+4 q_{3} \operatorname{Tr} S^{(1)} d t \\
= & \frac{16 \mu_{2}}{15} \operatorname{Tr} S^{(1)} \operatorname{Tr} S^{(2)} d t+\frac{8 \mu_{4}}{35} \operatorname{Tr} S^{(1)} d t .
\end{aligned}
$$

This completes the proof.

Theorem 2.9. The process $\left(\operatorname{Tr} S_{t}^{(1)}, \operatorname{Tr} S_{t}^{(2)}\right)$ is a recurrent diffusion with generator

$$
\begin{aligned}
L f(\kappa, m) & =\frac{1}{2}\left(\frac{\mu_{2}}{15}\left(14 \kappa^{2}-24 m\right)+\frac{16 \mu_{4}}{35}\right) \frac{\partial^{2} f}{\partial \kappa^{2}}(\kappa, m) \\
& +\left(\frac{16 \mu_{2}}{15} \kappa m+\frac{8 \mu_{4}}{35} \kappa\right) \frac{\partial^{2} f}{\partial \kappa \partial m}(\kappa, m) \\
& +\frac{1}{2}\left(\frac{32 \mu_{2}}{15} m^{2}+\frac{2 \mu_{4}}{35}\left(3 \kappa^{2}-4 m\right)\right) \frac{\partial^{2} f}{\partial m^{2}}(\kappa, m) \\
& +\frac{4 \mu_{2}}{15} \kappa \frac{\partial f}{\partial \kappa}(\kappa, m)+\frac{4 \mu_{2}}{15} m \frac{\partial f}{\partial m}(\kappa, m) .
\end{aligned}
$$

This diffusion never enters the region $\kappa^{2} \leq 4 m$ at strictly positive times a.s. . 


\section{Remarks.}

(1) In $n=3$, the three Lyapunov exponents are (see LeJan 1986) $\gamma_{1}=3, \gamma_{2}=0$, $\gamma_{3}=-3$. Thus there is always a stretching direction, a neutral direction and a compressing direction. A curve will always "see" the stretching direction. This roughly explains the positive recurrence of the curvature of a curve proved in LeJan 1986. In the case of a surface, the tangent plane will "see" the stretching and the neutral direction. This also explains recurrence. The authors plan a complete account of the situation in higher dimensions in a forthcoming paper.

(2) Since $X=\operatorname{Tr} S^{(1)}=\lambda_{1}+\lambda_{2}$ and $Y=\operatorname{Tr} S^{(2)}=\lambda_{1} \lambda_{2}$, it follows that $(X, Y)$ stays in the region $\kappa^{2} \geq 4 \mathrm{~m}$. The boundary of this region corresponds to $\lambda_{1}=\lambda_{2}$. That is $\kappa^{2}=4 m$ when the surface in question has an umbilic (i.e. a point where $\lambda_{1}=\lambda_{2}$ ). Thus, $x$ may be an umbilic for $M$ but $x_{t}, t>0$, will never be an umbilic for $M_{t}$.

One easily checks that the matrix $a$ appearing in the generator for this diffusion ( $2^{\text {nd }}$ order derivative coefficients) degenerates on the curve $\kappa^{2}-$ $4 m=0$. Moreover, the two eigenvectors of this matrix are, respectively, perpendicular to and tangent to the curve $\kappa^{2}-4 m=0$. The eigenvector perpendicular to this curve has eigenvalue zero. Notice the drift vector $\left(\frac{4 \mu_{2}}{15} \kappa, \frac{4 \mu_{2}}{15} m\right)$ points away from the region $\kappa^{2}-4 m<0$. Thus, when the point $x$ on the initial manifold $M$ is an umbilic point, the diffusion $\left(X_{t}, Y_{t}\right)$ has diffusion component only tangential the curve $\kappa^{2}=4 m$ and drifts away from this curve never to return.

(3) Neither $\operatorname{Tr} S^{(1)}$ nor $\operatorname{Tr} S^{(2)}$ is a diffusion by itself.

(4) In the case $n=3$ one can assert that $\left(X_{t}, Y_{t}\right)$ does not spend a lot of time in the region $\lambda=\left\{(\kappa, m): \kappa>M_{1}, m>M_{2}\right\}$ with $M_{1}$ and $M_{2}$ large positive constants. Recall the following results of LeJan (1986): draw a curve $\gamma$ on the initial manifold $M$ with $\gamma(0)=x$. Set $\gamma_{t}=\Phi_{t}(\gamma)$. Then the curvature $\tau_{t}$ of $\gamma_{t}$ at $\gamma_{t}(0)$ is a positive recurrent diffusion. Thus, the average amount of time that $\tau_{t}$ spends above a large positive constant is small. Since it is impossible to draw a curve on $M_{t}$ with zero curvature (or even small curvature) when $\left(X_{t}, Y_{t}\right) \in \Lambda,\left(X_{t}, Y_{t}\right)$ can not stay for long in $\Lambda$. More precise statements can be made by referring to the article of LeJan where the exact form of the invariant measure for $\tau_{t}$ is given.

Proof (of Theorem 2.9). The claim that $\left(X_{t}, Y_{t}\right)$ is a diffusion follows immediately from Theorem 2.8. Define $h=\sqrt{\kappa^{2}-4 m}$. Then with $a=\frac{\mu_{2}}{15}, b=\frac{\mu_{4}}{35}$, in $(m, h)$ coordinates

$$
\begin{aligned}
L f(\kappa, h) & =\left(4 a \kappa^{2}+3 a h^{2}+8 b\right) \frac{\partial^{2} f}{\partial \kappa^{2}}+14 a \kappa h \frac{\partial^{2} f}{\partial \kappa \partial h} \\
& +\left(3 a \kappa^{2}+4 a h^{2}+4 b\right) \frac{\partial^{2} f}{\partial h^{2}} \\
& +4 a \kappa \frac{\partial f}{\partial \kappa}+h^{-1}\left(3 a \kappa^{2}+a h^{2}+4 b\right) \frac{\partial f}{\partial h} .
\end{aligned}
$$


We prove the claim regarding lack of umbilics first as it eases a technical point which might arise in the proof of the recurrence. For this, it suffices to show that $H_{t}$ does not hit zero a.s. where $\left(X_{t}, H_{t}\right)$ is diffusion given in $(\kappa, h)$ coordinates. First define $\eta_{t}=3 a X_{t}^{2}+4 a H_{t}^{2}+4 b$ and then $\tau_{t}=\int_{0}^{t} \frac{d x}{\eta s}$. Then

$$
\begin{aligned}
H_{\tau_{t}} & =h+b_{t}+\int_{0}^{\tau_{t}} H_{s}^{-1}\left(3 a X_{s}^{2}+a H_{s}^{2}+4 b\right) d s \\
& =h+b_{t}+\int_{0}^{t} H_{\tau_{s}}^{-1}\left(3 a X_{\tau_{s}}^{2}+a H_{\tau_{s}}^{2}+4 b\right) \eta_{\tau_{s}}^{-1} d s
\end{aligned}
$$

with $b_{t}$ a one-dimensional Brownian motion. Fix now an $\epsilon \in(0,1 / 6)$ and suppose $0<h<1 / 6$ as well. Set

$$
\rho_{t}^{\epsilon}=h+b_{t}+\left(\frac{1}{2}+\epsilon\right) \int_{0}^{t} \frac{d s}{\rho_{s}^{\epsilon}} .
$$

If $\sigma_{\epsilon}=\inf \left\{t>0: H_{\tau_{t}}>2 \sqrt{\frac{\left(\frac{1}{2}-\epsilon\right) b}{(1+4 \epsilon) a}}\right\}$, then since for $h \leq \sqrt{\frac{\left(\frac{1}{2}-\epsilon\right) b}{(1+4 \epsilon) a}}$, one has $\left(\frac{1}{2}+\epsilon\right)<\frac{3 a \kappa^{2}+a h^{2}+4 b}{3 a \kappa^{2}+4 a h^{2}+4 b}$ for all $\kappa$. By an elementary comparison theorem (see IkedaWatanabe, 1989), it follows that $\rho_{t}^{\epsilon} \leq H_{\tau_{t}}$ for $t<\sigma_{\epsilon}$. Since $\rho_{t}^{\epsilon}$ can not hit zero (it is a Bessel process above the critical dimension), $H_{t}$ can't hit zero either.

For recurrence, we shall show $\left(X_{t}, H_{t}\right)$ is recurrent which will suffice. Set $f(\kappa, h)=\ln \left(\kappa^{6}+h^{6}\right)$. Then

$$
\begin{aligned}
f_{\kappa} & =\frac{6 \kappa^{5}}{\kappa^{6}+h^{6}}, \quad f_{h}=\frac{6 h^{5}}{\kappa^{6}+h^{6}} \\
f_{\kappa \kappa} & =\frac{30 \kappa^{4} h^{6}-6 \kappa^{10}}{\left(\kappa^{6}+h^{6}\right)^{2}}, \quad f_{\kappa h}=-\frac{36 \kappa^{5} h^{5}}{\left(\kappa^{6}+h^{6}\right)^{2}} ; \quad f_{h h}=\frac{30 \kappa^{6} h^{4}-6 h^{10}}{\left(\kappa^{6}+h^{6}\right)^{2}}
\end{aligned}
$$

and

$$
\begin{aligned}
L f=6 & \left(\kappa^{6}+h^{6}\right)^{-2}\left[\left(4 a \kappa^{2}+3 a h^{2}+8 b\right)\left(5 \kappa^{4} h^{6}-\kappa^{10}\right)-84 a \kappa^{6} h^{6}\right. \\
& +\left(3 a \kappa^{2}+4 a h^{2}+4 b\right)\left(5 \kappa^{6} h^{4}-h^{10}\right)+4 a \kappa^{12}+4 a \kappa^{6} h^{6} \\
& \left.+h^{4}\left(3 a \kappa^{2}+a h^{2}+4 b\right)\left(\kappa^{6}+h^{6}\right)\right] \\
=6 & \left(\kappa^{6}+h^{6}\right)^{-2}\left[-a\left(3 \kappa^{10} h^{2}-18 \kappa^{8} h^{4}+39 \kappa^{6} h^{6}-15 \kappa^{4} h^{8}+3 h^{12}\right)\right. \\
& \left.\quad-b\left(8 \kappa^{10}-24 \kappa^{6} h^{4}-40 \kappa^{4} h^{6}\right)\right] \\
& \kappa=u h \\
=- & 6\left(\kappa^{6}+h^{6}\right)^{-2} h^{10}\left[3 a h^{2}\left(u^{10}-6 u^{8}+13 u^{6}-5 u^{4}+1\right)\right. \\
& \left.+8 b u^{4}\left(u^{6}-3 u^{2}-5\right)\right]
\end{aligned}
$$

The polynomial $P(u)=u^{10}-6 u^{8}+13 u^{6}-5 u^{4}+1>0$ for all $u$. 
Also, there is a $u_{0}>0$ such that $Q(u)=u^{6}-3 u^{2}-5 \geq 0$ whenever $|u| \geq u_{0}$. Thus,

$$
L f=-6\left(\kappa^{6}+h^{6}\right)^{-2} h^{10}\left[3 a h^{2} P(u)+8 b u^{4} Q(u)\right]
$$

has

$$
\{L f \leq 0\} \supseteq\left\{(\kappa, h):|\kappa| \geq u_{0} h\right\} .
$$

Also, denote $M=\sup _{u} \frac{Q(u)}{P(u)}$, one sees

$$
\{L f \leq 0\} \supseteq\left\{(\kappa, h): h \geq \sqrt{\frac{86 M}{3 a}}\right\}
$$

Consequently, off of the compact triangle

$$
T=\left\{(\kappa, h):|\kappa| \leq u_{0} h\right\} \cap\left\{(\kappa, h): h \leq \sqrt{\frac{86 M}{3 a}}\right\}
$$

we have $L f \leq 0$. Now, define

$$
C(r)=\left\{(\kappa, h): h>0, \kappa^{6}+h^{6}<r\right\}
$$

and select $r_{0}$ large enough so that $C\left(r_{0}\right) \supset T$. Put for $R>r_{0}$

$$
u_{R}(\kappa, h)=\frac{\ln \left(\kappa^{6}+h^{6}\right)-\ln R}{\ell n r_{0}-\ell n R}
$$

Then on $C(R) \backslash C\left(r_{0}\right), L u_{R} \geq 0$ and $\left.u_{R}\right|_{\partial C\left(r_{0}\right) \cap\{h>0\}}=1,\left.u_{R}\right|_{\partial C(R) \cap\{h>0\}}=0$. Thus, if $\tau_{r}=\inf \left\{t>0:\left(X_{t}, H_{t}\right) \in \partial C(r)\right\}$ (recall, $H_{t}$ never hits 0 ), then as $u_{R}$ is $L$-subharmonic and has the same boundary values as $P_{(\kappa, w)}\left(\tau_{r_{0}}<T_{R}\right)$

$$
u_{R}(\kappa, h) \leq P_{(\kappa, h)}\left(\tau_{r_{0}}<\tau_{R}\right) .
$$

But $\lim _{R \uparrow \infty} u_{R}(\kappa, h)=1$ which implies the recurrence of $\left(X_{t}, H_{t}\right)$ and therefore the recurrence of $\left(X_{t}, Y_{t}\right)$. 


\section{REFERENCES}

P. Baxendale, (1984), Brownian motions in the diffeomorphism Group. I, Compositio Math. 53, 19-50.

P. Baxendale and T.Harris, (1986), Isotropic stochastic flows, Annals of Prob., Vol 14, 1155-1179.

R. Bishop and S. Goldberg, (1980), Tensor Analysis on Manifolds, Dover, Toronto.

Hardy, Littlewood, Polya, (1973), Inequalities, Cambridge University Press, Cambridge.

T. Harris, (1981), Brownian motions on the homeomorphisms of the plane, Annals of Prob. Vol. 9, 232-254.

N. Ikeda, S. Watanabe, (1989), Stochastic Differential Equations and Diffusion Processes, North-Holland, New York.

Y. LeJan, (1985), On isotropic Brownian motions, Zeit. für Wahr., 70, 609-620.

Y. LeJan, (1991), Asymototic properties of isotropic Brownian flows, in Spatial Stochastic Processes, ed. K. Alexander, J. Watkins, Birkhäuser, Boston, 219-232.

Y. LeJan, S. Watanabe (1984), Stochastic flows of diffeomorphisms, Proc. of the Taniguchi Symposium, 307-332, North Holland, New York.

M. Spivak, (1979), A comprehensive introduction to differential geometry, Publish or Perish, Berkeley. 


\section{Addendum. Higher dimensional hypersurfaces.}

Recall $\alpha_{\vec{\ell}}=u_{\ell_{1}} \wedge \cdots \wedge u_{\ell_{k}}, \alpha_{\vec{m}}=u_{m_{1}} \wedge \cdots \wedge u_{m_{k}}$, for $\vec{\ell}, \vec{m} \in I_{k}$. Define

$$
\alpha_{\vec{\ell}_{p}}=(-1)^{p+1} u_{\ell_{1}} \wedge \cdots \wedge \hat{u}_{\ell_{p}} \wedge \cdots \wedge u_{\ell_{k}}, \quad \alpha_{\vec{m}_{i}}=(-1)^{i+1} u_{m_{1}} \wedge \cdots \wedge \hat{u}_{m_{i}} \wedge \cdots \wedge u_{m_{k}} .
$$

Then $\alpha_{\vec{\ell}_{p}}=(-1)^{p+1} u_{\ell_{1}}^{(p)} \wedge \cdots \wedge u_{\ell_{k}-1}^{p} \quad \alpha_{\vec{m}_{i}}^{(i)}=(-1)^{p+1} u_{m_{1}}^{(i)} \wedge \cdots \wedge u_{m_{k-1}}^{(i)}$ defines $u_{\ell}^{(p)}$ and $u_{m}^{(i)}$, and set $S^{(0)}(\cdot, \cdot) \equiv 1$.

\section{Theorem A.1.}

$$
\begin{aligned}
d S^{(k)}\left(\alpha_{\vec{\ell}}, \alpha_{\vec{m}}\right) & =\sum_{i, p=1}^{k} S^{(k-1)}\left(\alpha_{\vec{\ell}_{p}}, \alpha_{\vec{m}_{i}}\right)\left\langle d B\left(u_{\ell_{p}}, u_{m_{i}}\right), \nu\right\rangle \\
& +k S^{(k)}\left(\alpha_{\vec{\ell}}, \alpha_{\vec{m}}\right) d W_{n}^{n}-\frac{k(n-k)(n-1) \mu_{2}}{2 n(n+2)} S^{(k)}\left(\alpha_{\vec{\ell}}, \alpha_{\vec{m}}\right) d t
\end{aligned}
$$

or, in a more synthetic form

$$
d S_{(\alpha)}^{(k)}=S^{(k-1)} \wedge d B^{\nu}(\alpha)+k S^{(k)}(\alpha)\left\langle d\langle W \nu, \nu\rangle-\frac{-k(n-k)(n-1)}{2 n(n+2)} S^{(k)}(\alpha),\right.
$$

with $\left\langle B^{\nu} u, v\right\rangle=\langle B(u, v), \nu\rangle$.

Proof. By Theorem 2.7 and Itô's formula,

$$
\begin{aligned}
& d S^{(k)}\left(\alpha_{\vec{\ell}}, \alpha_{\vec{m}}\right)=\sum_{\sigma \in S_{k}}(-1)^{\sigma} \sum_{i=1}^{k}\left[\prod_{\substack{j=1 \\
j \neq i}}^{k}\left\langle S\left(u_{\ell_{\sigma(j)}}, u_{m_{j}}\right), \nu\right\rangle\right]\left\langle d B\left(u_{\ell_{\sigma(i)}}, u_{m_{i}}\right), \nu\right\rangle \\
&+k S^{(k)}\left(\alpha_{\vec{\ell}}, \alpha_{\vec{m}}\right) d W_{n}^{n}-\frac{k(n-1)(n-1) \mu_{2}}{2 n(n+2)} S^{(k)}\left(\alpha_{\vec{\ell}}, \alpha_{\vec{m}}\right) d t \\
&+\frac{1}{2} \sum_{\sigma \in S_{k}}(-1)^{\sigma} \sum_{1 \leq i \neq p \leq k}\left[\prod_{\substack{j=1 \\
j \neq\{i, p\}}}^{k}\left\langle S\left(u_{\ell_{\sigma(j)}}, u_{m_{j}}\right), \nu\right\rangle\right]\left[d B^{n}\left(u_{\ell_{\sigma(i)}}, u_{m_{i}}\right), d B^{n}\left(u_{\ell_{\sigma(p)}}, u_{m_{p}}\right)\right\rangle \\
&\left.+\left\langle d B^{n}\left(u_{\ell_{\sigma(i)}}, u_{m_{p}}\right), d B^{n}\left(u_{\ell_{\sigma(p)}}, u_{m_{i}}\right)\right\rangle+\left\langle d B^{n}\left(u_{\ell_{\sigma(i)}}, u_{\ell_{\sigma(p)}}\right), d B^{n}\left(u_{m_{i}}, u_{m_{p}}\right)\right\rangle\right] \\
&+\frac{k(k-1)(n-1) \mu_{2}}{2 n(n+2)} S^{(k)}\left(\alpha_{\vec{\ell}}, \alpha_{\vec{m}}\right) d t .
\end{aligned}
$$

The first term may be written

$$
\begin{gathered}
\sum_{i, p=1}^{k} \sum_{\substack{\sigma \in S_{k} \\
\sigma(i)=p}}(-1)^{\sigma}\left[\prod_{\substack{j=1 \\
j \neq i}}^{k}\left\langle S\left(u_{\ell_{\sigma(j)}}, u_{m_{j}}\right), \nu\right\rangle\right]\left\langle d B\left(u_{\ell_{p}}, u_{m_{i}}\right), \nu\right\rangle \\
=\sum_{i, p=1}^{k}\left[\sum_{\sigma=S_{k-1}}(-1)^{p+i}(-1)^{\sigma}\left[\prod_{\substack{j=1 \\
j \neq i}}^{k}\left\langle S\left(u_{\ell_{\sigma(j)}}^{(p)}, u_{m_{j}}^{(i)}\right), \nu\right\rangle\right]\right]\left\langle d B\left(u_{\ell_{p}}, u_{m_{i}}\right), \nu\right\rangle \\
=\sum_{i, p=1}^{k} S^{(k-1)}\left(\alpha_{\vec{\ell}_{p}}, \alpha_{\vec{m}_{i}}\right)\left\langle d B\left(u_{\ell_{p}}, u_{m_{i}}\right), \nu\right\rangle .
\end{gathered}
$$


The third term combines with the fifth term to give

$$
-\frac{k(n-k)(n-1) \mu_{2}}{2 n(n+2)} S^{(k)}\left(\alpha_{\vec{\ell}}, \alpha_{\vec{m}}\right) d t .
$$

Finally, in the fourth term, notice that given $\sigma \in S_{k}$ and $i, p \in\{1, \ldots, k\}$ there is exactly one other $\eta \in S_{k}$ such that both $\{\sigma(i), \sigma(p)\}=\{\eta(i), \eta(p)\}$ and $\sigma(j)=\eta(j)$ for $j \notin\{i, p\}$. Furthermore, $(-1)^{\sigma}=-(-1)^{\eta}$, and

$$
\begin{aligned}
& {\left[\prod_{\substack{j=1 \\
j \notin\{i, p\}}}^{k}\left\langle S\left(u_{\ell_{\sigma(j)}}, u_{m_{j}}\right), \nu\right\rangle\right]\left[\left\langle B^{n}\left(u_{\ell_{\sigma(i)}}, u_{m_{i}}\right), d B^{n}\left(u_{\ell_{\sigma(p)}}, u_{m_{p}}\right)\right\rangle\right.} \\
& \left.\quad+\left\langle d B^{n}\left(u_{\ell_{\sigma(i)}}, u_{m_{p}}\right), d B^{n}\left(u_{\ell_{\sigma(p)}}, u_{m_{i}}\right)\right\rangle+\left\langle d B^{n}\left(u_{\ell_{\sigma(i)}}, u_{\ell_{\sigma(p)}}\right), d B^{n}\left(u_{m_{i}}, u_{m_{p}}\right)\right\rangle\right] \\
& =\left[\prod_{\substack{j=1 \\
j \notin\{i, p\}}}^{k}\left\langle S\left(u_{\ell_{\eta(j)}}, u_{m_{j}}\right), \nu\right\rangle\right]\left[\left\langle d B^{n}\left(u_{\ell_{\eta(i)}}, u_{m_{i}}\right), d B^{n}\left(u_{\ell_{\eta(p)}}, u_{m_{p}}\right)\right\rangle\right. \\
& + \\
& \quad\left\langle d B^{n}\left(u_{\ell_{\eta(i)}}, u_{m_{p}}\right), d B^{n}\left(u_{\ell_{\eta(p)}}, u_{m_{i}}\right)\right\rangle \\
& \left.\quad+\left\langle d B^{n}\left(u_{\ell_{\eta(i)}}, u_{\left.\ell_{\eta(p)}\right)}\right), d B^{n}\left(u_{m_{i}}, u_{m_{p}}\right)\right\rangle\right]
\end{aligned}
$$

Thus exchanging the sums in the fourth term reveals the cancellation of terms from these $\sigma-\eta$ pairs and so the whole term vanishes. This finishes the proof.

From LeJan (1985) we recall the notation

$$
\tau_{\ell}^{j} \beta=e^{j} \wedge i\left(e^{\ell}\right) \beta
$$

where $i\left(e^{\ell}\right) \beta=\Sigma(-1)^{k+1}\left\langle\beta_{k}, e^{\ell}\right\rangle \beta_{1} \wedge \cdots \wedge \hat{\beta}_{k} \wedge \cdots \wedge \beta_{m}$. The situation for general $n$ is given by the following Theorem which we prove in the Addendum.

Theorem A.2. For $1 \leq k \leq n-1$,

$$
\begin{gathered}
d \operatorname{Tr} S^{(k)}=\left[\sum_{i, p=1}^{k} S^{(k-1)}\left(\alpha_{\vec{\ell}_{p}}, \alpha_{\vec{m}_{i}}\right)\left\langle d B\left(u_{\ell_{p}}, u_{m_{i}}\right), \nu\right\rangle\right]\left\langle\alpha^{\vec{\ell}}, \alpha^{\vec{m}}\right\rangle\|\alpha\|^{-2} \\
+\operatorname{Tr} S^{(k)}\left[k d W_{n}^{n}-2 \sum_{i=1}^{n-1} d W_{i}^{i}\right] \\
+S^{(k)}\left(\alpha_{\vec{\ell}}, \alpha_{\vec{m}}\right)\left(\left\langle\tau_{i}^{j} \alpha^{\vec{\ell}}, \alpha^{\vec{m}}\right\rangle+\left\langle\alpha^{\vec{\ell}}, \tau_{i}^{j} \alpha^{\vec{m}}\right\rangle\right) d W_{j}^{i}\|\alpha\|^{-2} \\
\quad-\frac{(n+1) k(n-k) \mu_{2}}{2 n(n+2)} \operatorname{Tr} S^{(k)} d t, \text { or } \\
d T r S^{(k)}=d\left\langle S^{(k)} \alpha, \alpha\right\rangle /\|\alpha\|^{2} \\
=\left\langle S^{(k-1)} \wedge d B^{\nu}(\alpha)+\left(\langle d W \nu, \nu\rangle-2 \frac{\langle d W \alpha, \alpha\rangle}{\|\alpha\|^{2}}\right) S^{(k)}(\alpha)\right. \\
\left.+S^{(k)} \wedge d W(\alpha), \alpha\right\rangle /\|\alpha\|^{2}-\frac{(n+1) k(n-k) \mu_{2}}{2 n(n+2)}\left\langle S^{(k)}(\alpha), \alpha\right\rangle /\|\alpha\|^{2} .
\end{gathered}
$$


Proof. For $\vec{\ell} \in I_{k}$,

$$
\begin{aligned}
d\left\langle\alpha^{\vec{\ell}}, \alpha^{\vec{m}}\right\rangle & =\left(\left\langle\tau_{i}^{j} \alpha^{\vec{\ell}}, \alpha^{\vec{m}}\right\rangle+\left\langle\alpha^{\vec{\ell}}, \tau_{i}^{j} \alpha^{\vec{m}}\right\rangle\right) d W_{j}^{i} \\
& +\frac{(k+1)(n-1-k) \mu_{2}}{n}\left\langle\alpha^{\vec{\ell}}, \alpha^{\vec{m}}\right\rangle d t
\end{aligned}
$$

$$
\frac{d\|\alpha\|^{-2}}{\|\alpha\|^{-2}}=-2 \sum_{i=1}^{n-1} d W_{i}^{i}-\frac{(n-2)(n-1) \mu_{2}}{n(n+2)} d t
$$

Since

$$
d\|\alpha\|^{-2}=-2\|\alpha\|^{-2} d W_{i}^{i}+A_{t}, \quad A_{t} \text { has bounded variation }
$$

$$
T_{r} S^{(k)}=S^{(k)}\left(\alpha_{\vec{\ell}}, \alpha_{\vec{m}}\right)\left\langle\alpha^{\vec{\ell}}, \alpha^{\vec{m}}\right\rangle\|\alpha\|^{-2}
$$

by Itô's formula,

$$
\begin{aligned}
d \operatorname{Tr} S^{(k)} & =\left(d S^{(k)}\left(\alpha_{\vec{\ell}}, \alpha_{\vec{m}}\right)\right)\left\langle\alpha^{\vec{\ell}}, \alpha^{\vec{m}}\right\rangle\|\alpha\|^{-2} \\
& +S^{(k)}\left(\alpha_{\vec{\ell}}, \alpha_{\vec{m}}\right)\left(d\left\langle\alpha^{\vec{\ell}}, \alpha^{\vec{m}}\right\rangle\right)\|\alpha\|^{-2} \\
& +\operatorname{Tr} S^{(k)} \frac{d\|\alpha\|^{-2}}{\|\alpha\|^{-2}} \\
& +\left\langle d S^{(k)}\left(\alpha_{\vec{\ell}}, \alpha_{\vec{m}}\right), d\left\langle\alpha^{\vec{\ell}}, \alpha^{\vec{m}}\right\rangle\right\rangle\|\alpha\|^{-2} \\
& +\left\langle d S^{(k)}\left(\alpha_{\vec{\ell}}, \alpha_{\vec{m}}\right), d\|\alpha\|^{-2}\right\rangle\left\langle\alpha^{\vec{\ell}}, \alpha^{\vec{m}}\right\rangle \\
& +S^{(k)}\left(\alpha_{\vec{\ell}}, \alpha_{\vec{m}}\right)\left\langle d\left\langle\alpha^{\vec{\ell}}, \alpha^{\vec{m}}\right\rangle, d\|\alpha\|^{-2}\right\rangle
\end{aligned}
$$

Proceeding in order through the terms, we have using Theorem A.1

$$
\begin{aligned}
& d S^{(k)}\left(\alpha_{\vec{\ell}}, \alpha_{\vec{m}}\right)\left\langle\alpha^{\vec{\ell}}, \alpha^{\vec{m}}\right\rangle\|\alpha\|^{-2} \\
& =\left[\sum_{i, p=1}^{k} \mathcal{S}^{(k-1)}\left(\alpha_{\vec{\ell}_{p}}, \alpha_{\vec{m}_{i}}\right)\left\langle d B\left(u_{\ell_{p}}, u_{m_{i}}\right), \nu\right\rangle\right]\left\langle\alpha^{\vec{\ell}}, \alpha^{\vec{m}}\right\rangle\|\alpha\|^{-2} \\
& +k \operatorname{Tr} \mathcal{S}^{(k)} d W_{n}^{n}-\frac{k(2 n-k)(n-1) \mu_{2}}{2 n(n+2)} \operatorname{Tr} S^{(k)} d t .
\end{aligned}
$$

From (A.1) follows,

$$
\begin{aligned}
& S^{(k)}\left(\alpha_{\vec{\ell}}, \alpha_{\vec{m}}\right) d\left\langle\alpha^{\vec{\ell}}, \alpha^{\vec{m}}\right\rangle|| \alpha \|^{-2} \\
& =S^{(k)}\left(\alpha_{\vec{\ell}}, \alpha_{\vec{m}}\right)\left(\left\langle\tau_{i}^{j} \alpha^{\vec{\ell}}, \alpha^{\vec{m}}\right\rangle+\left\langle\alpha^{\vec{\ell}}, \tau_{i}^{j} \alpha^{\vec{m}}\right\rangle\right) d W_{j}^{i}\|\alpha\|^{-2} \\
& +\frac{(k+1)(n-k-1) \mu_{2}}{n} \operatorname{Tr} S^{(k)} d t .
\end{aligned}
$$


By (A.2)

$$
\operatorname{Tr} S^{(k)} \frac{d\|\alpha\|^{-2}}{\|\alpha\|^{-2}}=-2 \operatorname{Tr} S^{(k)} \sum_{i=1}^{n-1} d W_{i}^{i}-\frac{(n-2)(n-1) \mu_{2}}{n(n+2)} \operatorname{Tr} S^{(k)} d t .
$$

Combining Theorem A.1 and (A.1),

$$
\begin{aligned}
\left\langle d S^{(k)}\right. & \left.\left(\alpha_{\vec{\ell}}, \alpha_{\vec{m}}\right), d\left\langle\alpha^{\vec{\ell}}, \alpha^{\vec{m}}\right\rangle\right\rangle\|\alpha\|^{-2} \\
& =k S^{(k)}\left(\alpha_{\vec{\ell}}, \alpha_{\vec{m}}\right)\left(\left\langle\tau_{i}^{j} \alpha^{\vec{\ell}}, \alpha^{\vec{m}}\right\rangle+\left\langle\alpha^{\vec{\ell}}, \tau_{i}^{j} \alpha^{\vec{m}}\right\rangle\right)\left\langle d W_{j}^{i}, d W_{n}^{n}\right\rangle \\
& =\frac{k \mu_{2}}{n(n+2)} S^{(k)}\left(\alpha_{\vec{\ell}}, \alpha_{\vec{m}}\right)\left(\left\langle\tau_{i}^{j} \alpha^{\vec{\ell}}, \alpha^{\vec{m}}\right\rangle+\left\langle\alpha^{\vec{\ell}}, \tau_{i}^{j} \alpha^{\vec{m}}\right\rangle\right)\left[(n+1) \delta_{n}^{i} \delta_{n}^{j}-\delta_{j}^{i} \delta_{n}^{n}-\delta_{n}^{i} \delta_{n}^{j}\right] d t \\
& =-\frac{k \mu_{2}}{n(n+2)} S^{(k)}\left(\alpha_{\vec{\ell}}, \alpha_{\vec{m}}\right)\left(\left\langle\tau_{i}^{i} \alpha^{\vec{\ell}}, \alpha^{\vec{m}}\right\rangle+\left\langle\alpha^{\vec{\ell}}, \tau_{i}^{i} \alpha^{\vec{m}}\right\rangle\right) d t \\
& =-\frac{2 k(n-k-1) \mu_{2}}{n(n+2)} \operatorname{Tr} S^{(k)} d t .
\end{aligned}
$$

By Theorem A.1 and (A.3)

$$
\begin{aligned}
\left\langle d S^{(k)}\right. & \left.\left(\alpha_{\vec{\ell}}, \alpha_{\vec{m}}\right), d\|\alpha\|^{-2}\right\rangle\left\langle\alpha^{\vec{\ell}}, \alpha^{\vec{m}}\right\rangle \\
& =-2 k S^{(k)}\left(\alpha_{\vec{\ell}}, \alpha_{\vec{m}}\right)\left\langle d W_{n}^{n}, d W_{i}^{i}\right\rangle\left\langle\alpha^{\vec{\ell}}, \alpha^{\vec{m}}\right\rangle\|\alpha\|^{-2} \\
& =\frac{2 k(n-1) \mu_{2}}{n(n+2)} \operatorname{Tr} S^{(k)} d t .
\end{aligned}
$$

From (A.1) and (A.3) we get

$$
\begin{aligned}
S^{(k)} & \left(\alpha_{\vec{\ell}}, \alpha_{\vec{m}}\right)\left\langle d\left\langle\alpha^{\vec{\ell}}, \alpha^{\vec{m}}\right\rangle, d\|\alpha\|^{-2}\right\rangle \\
& =-2 S^{(k)}\left(\alpha_{\vec{\ell}}, \alpha_{\vec{m}}\right)\left(\left\langle\tau_{i}^{j} \alpha^{\vec{\ell}}, \alpha^{\vec{m}}\right\rangle+\left\langle\alpha^{\vec{\ell}}, \tau_{i}^{j} \alpha^{\vec{m}}\right\rangle\right)\left\langle d W_{p}^{p}, d W_{j}^{i}\right\rangle\|\alpha\|^{-2} \\
& =\frac{2 \mu_{2}}{n(n+2)} \mathcal{S}^{(k)}\left(\alpha_{\vec{\ell}}, \alpha_{\vec{m}}\right)\left(\left\langle\tau_{i}^{i} \alpha^{\vec{\ell}}, \alpha^{\vec{m}}\right\rangle+\left\langle\alpha^{\vec{\ell}}, \tau_{i}^{i} \alpha^{\vec{m}}\right\rangle\right)\|\alpha\|^{-2} d t \\
& =-\frac{4(n-k-1) \mu_{2}}{n(n+2)} \operatorname{Tr} S^{(k)} d t
\end{aligned}
$$

Summing (A.4)-(A.8) shows

$$
\begin{aligned}
d T_{r} S^{(k)} & =\left[\sum_{i, p=1}^{k}(-1)^{i+p+1} \mathcal{S}^{(k-1)}\left(\alpha_{\vec{\ell}}^{\ell_{p}}, \alpha_{\vec{m}}^{m_{i}}\right)\left\langle d B\left(u_{\ell_{p}}, u_{m_{i}}\right), \nu\right\rangle\right]\left\langle\alpha^{\vec{\ell}}, \alpha^{\vec{m}}\right\rangle\|\alpha\|^{-2} \\
& +\operatorname{Tr} S^{(k)}\left[k d W_{n}^{n}-2 d W_{i}^{i}\right] \\
& +S^{(k)}\left(\alpha_{\vec{\ell}}, \alpha_{\vec{m}}\right)\left(\left\langle\tau_{i}^{j} \alpha^{\vec{\ell}}, \alpha^{\vec{m}}\right\rangle+\left\langle\alpha^{\vec{\ell}}, \tau_{i}^{j} \alpha^{\vec{m}}\right\rangle\right) d W_{j}^{i} \cdot\|\alpha\|^{-2} \\
& +\frac{k(n-k)(n+1) \mu_{2}}{2 n(n+2)} \operatorname{Tr} S^{(k)} d t
\end{aligned}
$$

and the proof is complete. 
Theorem A.3. For $n-1 \geq k \geq \ell \geq 1$,

$$
\begin{aligned}
& \left\langle d \operatorname{Tr} S^{(k)}, d \operatorname{Tr} S^{(\ell)}\right\rangle=\frac{\mu_{2}}{n(n+2)}[(k \ell+2(k+\ell)+4)(n-1) \\
& -2(k+2)(n-\ell-1)-2(\ell+2)(n-k-1)-4(n-\ell-1)(n-k-1)] \operatorname{Tr} S^{(k)} \operatorname{Tr} S^{(\ell)} d t \\
& +\left(1-\delta_{n-1}^{k}\right) \frac{4 \mu_{2}}{(n+2)} \sum_{j=0}^{(n-k-1) \wedge \ell} d(n,(k, \ell) ; j) \operatorname{Tr} S^{(k+j)} \operatorname{Tr} S^{(\ell-j)} d t \\
& +C_{1122}^{n n} \sum_{j=0}^{(n-k) \wedge(\ell-1)} a(n,(k, \ell) ; j) \operatorname{Tr} S^{(k+j-1)} \operatorname{Tr} S^{(\ell-j-1)} d t
\end{aligned}
$$

where

$$
\begin{aligned}
d(n,(k, \ell) ; 0) & =(n-1-k) \\
d(n,(k, \ell) ; j) & =(n-1-k-j)\left(\begin{array}{c}
k-\ell+2 j \\
j
\end{array}\right)-\sum_{r=0}^{j-1} d(n,(k, \ell) ; r)\left(\begin{array}{c}
k-\ell+2 j \\
j-r
\end{array}\right), j \geq 1 \\
b(n,(k, \ell) ; 0) & =(n-k)(n-\ell+2) \\
b(n,(k, \ell) ; j) & =3(n-k-j)\left(\begin{array}{c}
k-\ell+2 j \\
j
\end{array}\right)+(k-\ell+2 j)(k-\ell+2 j-1)\left(\begin{array}{c}
k-\ell+2 j-2 \\
j-1
\end{array}\right) \\
& +(k-\ell+2 j)(n-k-j)\left[\left(\begin{array}{c}
k-\ell+2 j-1 \\
j
\end{array}\right)+\left(\begin{array}{c}
k-\ell+2 j-1 \\
j-1
\end{array}\right)\right] \\
& +(n-k-j)(n-k-j-1)\left(\begin{array}{c}
k-\ell+2 j \\
j
\end{array}\right), j \geq 1 \\
a(n,(k, \ell) ; 0) & =b(n,(k, \ell) ; 0) \\
a(n,(k, \ell) ; j) & =b(n,(k, \ell) ; j)-\sum_{m=0}^{j-1} a(n,(k, \ell) ; m)\left(\begin{array}{c}
k-\ell+2 j \\
j-m
\end{array}\right) \\
C_{1122}^{n n}\left(n^{2}-1\right) & =\frac{(n+3) \mu_{4}}{n(n+2)(n+4)} .
\end{aligned}
$$

Proof. We compute the quadratic variation terms in $\left\langle d \operatorname{Tr} S^{(k)}, d \operatorname{Tr} S^{(\ell)}\right\rangle, k \geq \ell$, which do not arise from $d B$. These are

$$
\begin{gathered}
\left\langle\operatorname{Tr} S^{(k)}\left[k d W_{n}^{n}-2 \sum_{i=1}^{n-1} d W_{i}^{i}\right]+S^{(k)}\left(\alpha_{\vec{\ell}}, \alpha_{\vec{m}}\right)\left(\left\langle\tau_{h}^{j} \alpha^{\vec{\ell}}, \alpha^{\vec{m}}\right\rangle+\left\langle\alpha^{\vec{\ell}}, \tau_{h}^{j} \alpha^{\vec{m}}\right\rangle\right)\|\alpha\|^{-2} d W_{j}^{h},\right. \\
\left.\operatorname{Tr} S^{(\ell)}\left[\ell d W_{n}^{n}-2 \sum_{m=1}^{n-1} d W_{m}^{m}\right]+S^{(\ell)}\left(\alpha_{\vec{r}}, \alpha_{\vec{s}}\right)\left(\left\langle\tau_{q}^{p} \alpha^{\vec{r}}, \alpha^{\vec{s}}\right\rangle+\left\langle\alpha^{\vec{r}}, \tau_{q}^{p} \alpha^{\vec{s}}\right\rangle\right)\|\alpha\|^{-2} d W_{p}^{q}\right\rangle
\end{gathered}
$$

unless $k=n-1$ or both $\ell=k=n-1$ in which case, the term $S^{(k)}\left(\alpha_{\vec{\ell}}, \alpha_{\vec{m}}\right)$ $\left(\left\langle\tau_{i}^{j} \alpha^{\vec{\ell}}, \alpha^{\vec{m}}\right\rangle+\left\langle\alpha^{\vec{\ell}}, \tau_{i}^{j} \alpha^{\vec{m}}\right\rangle\right)\|\alpha\|^{-2} d W_{j}^{i}=0$ or both this and the corresponding $S^{(\ell)}$ term vanishes. Thus we get

$$
\operatorname{Tr} S^{(k)} \operatorname{Tr} S^{(\ell)}\left\langle k d W_{n}^{n}-2 \sum_{i=1}^{n-1} d W_{i}^{i}, \ell d W_{n}^{n}-2 \sum_{j=1}^{n-1} d W_{j}^{j}\right\rangle
$$




$$
\begin{aligned}
& +\operatorname{Tr} S^{(k)} S^{(\ell)}\left(\alpha_{\vec{r}}, \alpha_{\vec{s}}\right)\left(\left\langle\tau_{q}^{p} \alpha^{\vec{r}}, \alpha^{\vec{s}}\right\rangle+\left\langle\alpha^{\vec{r}}, \tau_{q}^{p} \alpha^{\vec{s}}\right\rangle\right)\|\alpha\|^{-2}\left(1-\delta_{n-1}^{\ell}\right) \\
& \left\langle k d W_{n}^{n}-2 \sum_{i=1}^{n-1} d W_{i}^{i}, d W_{p}^{q}\right\rangle \\
& +\operatorname{Tr} S^{(\ell)} S^{(k)}\left(\alpha_{\vec{\ell}}, \alpha_{\vec{m}}\right)\left(\left\langle\tau_{h}^{j} \alpha^{\vec{\ell}}, \alpha^{\vec{m}}\right\rangle+\left\langle\alpha^{\vec{\ell}}, \tau_{h}^{j} \alpha^{\vec{m}}\right\rangle\right)\|\alpha\|^{-2}\left(1-\delta_{n-1}^{k}\right) \\
& \left\langle\ell d W_{n}^{n}-2 \sum_{i=1}^{n-1} d W_{i}^{i}, d W_{j}^{h}\right\rangle \\
& +S^{(k)}\left(\alpha_{\vec{\ell}}, \alpha_{\vec{m}}\right) S^{(\ell)}\left(\alpha_{\vec{r}}, \alpha_{\vec{s}}\right)\left(\left\langle\tau_{h}^{j} \alpha^{\vec{\ell}}, \alpha^{\vec{m}}\right\rangle+\left\langle\alpha^{\vec{\ell}}, \tau_{h}^{j}, \alpha^{\vec{m}}\right\rangle\right)\left(\left\langle\tau_{q}^{p} \alpha^{\vec{r}}, \alpha^{\vec{s}}\right\rangle\right. \\
& \left.\quad+\left\langle\alpha^{\vec{r}}, \tau_{q}^{p} \alpha^{\vec{s}}\right\rangle\right)\left(1-\delta_{n-1}^{\ell}\right) \\
& \cdot\left(1-\delta_{n-1}^{k}\right)\|\alpha\|^{-4}\left\langle d W_{j}^{h}, d W_{p}^{q}\right\rangle \\
& =\frac{\mu_{2}}{n(n+2)} \operatorname{Tr}^{(k)} \operatorname{Tr}^{(\ell)}[k \cdot \ell(n-1)+2(k+\ell)(n-1)+4(n-1)] d t \\
& +\frac{\mu_{2}}{n(n+2)} \operatorname{Tr}^{(k)} S^{(\ell)}\left(\alpha_{\vec{r}}, \alpha_{\vec{s}}\right)\left(\left\langle\tau_{p}^{p} \alpha^{\vec{r}}, \alpha^{\vec{s}}\right\rangle+\left\langle\alpha^{\vec{r}}, \tau_{p}^{p} \alpha^{\vec{s}}\right\rangle\right)\|\alpha\|^{-2}\left(1-\delta_{n-1}^{\ell}\right)\left[k C_{n p}^{n p}-2 \sum_{i=1}^{n-1} C_{i p}^{i p}\right] d t \\
& +\frac{\mu_{2}}{n(n+2)} \operatorname{Tr}^{(\ell)} S^{(k)}\left(\alpha_{\vec{\ell}}, \alpha_{\vec{m}}\right)\left(\left\langle\tau_{j}^{j} \alpha^{\vec{\ell}}, \alpha^{\vec{m}}\right\rangle+\left\langle\alpha^{\vec{\ell}}, \tau_{j}^{j} \alpha^{\vec{m}}\right\rangle\right)\|\alpha\|^{-2}\left(1-\delta_{n-1}^{k}\right)\left[\ell C_{n j}^{n j}-2 \sum_{i=1}^{n-1} C_{i j}^{i j}\right] d t \\
& +\frac{\mu_{2}}{n(n+2)} S^{(k)}\left(\alpha_{\vec{\ell}}, \alpha_{\vec{m}}\right) S^{(k)}\left(\alpha_{\vec{r}}, \alpha_{\vec{s}}\right)\left(\left\langle\tau_{h}^{j} \alpha^{\vec{\ell}}, \alpha^{\vec{m}}\right\rangle+\left\langle\alpha^{\vec{\ell}}, \tau_{h}^{j} \alpha^{\vec{m}}\right\rangle\right)\left(\left\langle\tau_{q}^{p} \alpha^{\vec{r}}, \alpha^{\vec{s}}\right\rangle\right. \\
& \left.+\left\langle\alpha^{\vec{r}}, \tau_{q}^{p} \alpha^{\vec{s}}\right\rangle\right)\left(1-\delta_{n-1}^{k}\right)\|\alpha\|^{-4}\left[(n+1) \delta_{q}^{h} \delta_{p}^{j}-\delta_{j}^{h} \delta_{p}^{q}-\delta_{p}^{h} \delta_{j}^{q}\right] d t
\end{aligned}
$$

We now proceed singly through the last three terms. Using the invariance of our expressions under a change of basis we may take the $u_{i}$ to be the unit principal curvature directions. Then

$$
\begin{aligned}
& S^{(k)}\left(\alpha_{\vec{\ell}}, \alpha_{\vec{m}}\right)=\lambda_{\vec{\ell}} \delta_{\vec{m}}^{\vec{\ell}} \\
& S^{(\ell)}\left(\alpha_{\vec{r}}, \alpha_{\vec{s}}\right)=\lambda_{\vec{r}} \delta_{\vec{s}}^{\vec{r}} \\
& \left\langle\tau_{q}^{p} \alpha^{\vec{r}}, \alpha^{\vec{s}}\right\rangle \delta_{\vec{s}}^{\vec{r}}=\delta_{q}^{p} \delta_{\vec{s}}^{\vec{r}} \text { if and only if } p \in \vec{r},=0 \quad \text { otherwise, } \\
& \left\langle\tau_{h}^{j} \alpha^{\vec{\ell}}, \alpha^{m}\right\rangle \delta_{\vec{m}}^{\vec{\ell}}=\delta_{h}^{j} \delta_{\vec{m}}^{\vec{\ell}} \text { if and only if } j \in \vec{\ell},=0 \quad \text { otherwise. }
\end{aligned}
$$

Thus,

$$
\begin{aligned}
& \frac{\mu_{2}}{n(n+2)} \operatorname{Tr} S^{(k)} S^{(\ell)}\left(\alpha_{\vec{r}}, \alpha_{\vec{s}}\right)\left(\left\langle\tau_{p}^{p} \alpha^{\vec{r}}, \alpha^{\vec{s}}\right\rangle+\left\langle\alpha^{\vec{r}}, \tau_{p}^{p} \alpha^{\vec{s}}\right\rangle\right)\left(1-\delta_{n-1}^{\ell}\right)\|\alpha\|^{-2}\left[k C_{n p}^{n p}-2 \sum_{i=1}^{n-1} C_{i p}^{i p}\right] d t \\
= & \frac{\mu_{2}}{n(n+2)} \operatorname{Tr} S^{(k)} \lambda_{\vec{r}}\left\langle\tau_{p}^{p} \alpha^{\vec{r}}, \alpha^{\vec{r}}\right\rangle\left(1-\delta_{n-1}^{\ell}\right)\left[k\left(n \delta_{p}^{n}-\delta_{n}^{n} \delta_{p}^{p}\right)-2\right] d t \\
= & -\frac{2(k+2)(n-\ell-1) \mu_{2}}{n(n+2)} \operatorname{Tr} S^{(k)} \operatorname{Tr} S^{(\ell)} d t .
\end{aligned}
$$

Similarly,

$$
\begin{aligned}
& \frac{\mu_{2}}{n(n+2)} \operatorname{Tr} S^{(\ell)} S^{(k)}\left(\alpha_{\vec{\ell}}, \alpha_{\vec{m}}\right)\left(\left\langle\tau_{j}^{j} \alpha^{\vec{\ell}}, \alpha^{\vec{m}}\right\rangle+\left\langle\alpha^{\vec{\ell}}, \tau_{j}^{j} \alpha^{\vec{m}}\right\rangle\right)\|\alpha\|^{-2}\left(1-\delta_{n-1}^{k}\right)\left(\ell C_{n j}^{n j}-2 \sum_{i=1}^{n-1} C_{i j}^{i j}\right) d t \\
= & \frac{-2(\ell+2)(n-k-1) \mu_{2}}{n(n+2)} \operatorname{Tr} S^{(k)} \operatorname{Tr} S^{(\ell)} d t .
\end{aligned}
$$


Finally, the last term is

$$
\begin{aligned}
& \left(1-\delta_{n-1}^{k}\right) \frac{4 \mu_{2}}{n(n+2)} \lambda_{\vec{\ell}} \lambda_{\vec{r}}\left\langle\tau_{h}^{j} \alpha^{\vec{\ell}}, \alpha^{\vec{\ell}}\right\rangle\left\langle\tau_{q}^{p} \alpha^{\vec{r}}, \alpha^{\vec{r}}\right\rangle\left(1-\delta_{n-1}^{\ell}\right)\left[(n+1) \delta_{q}^{h} \delta_{p}^{j}-\delta_{j}^{h} \delta_{p}^{q}-\delta_{p}^{h} \delta_{j}^{q}\right] d t \\
& =\left(1-\delta_{n-1}^{k}\right) \frac{4 \mu_{2}}{n(n+2)} \lambda_{\vec{\ell}} \lambda_{\vec{r}}\left[(n+1)\left\langle\tau_{h}^{j} \alpha^{\vec{\ell}}, \alpha^{\vec{\ell}}\right\rangle\left\langle\tau_{h}^{j} \alpha^{\vec{r}}, \alpha^{\vec{r}}\right\rangle-\left\langle\tau_{j}^{j} \alpha^{\vec{\ell}}, \alpha^{\vec{\ell}}\right\rangle\left\langle\tau_{p}^{p} \alpha^{\vec{r}}, \alpha^{\vec{r}}\right\rangle\right. \\
& \left.-\left\langle\tau_{h}^{j} \alpha^{\vec{\ell}}, \alpha^{\vec{\ell}}\right\rangle\left\langle\tau_{j}^{h} \alpha^{\vec{r}}, \alpha^{\vec{r}}\right\rangle\right] d t \\
& =\left(1-\delta_{n-1}^{k}\right) \frac{4 \mu_{2}}{n(n+2)} \lambda_{\vec{\ell}} \lambda_{\vec{r}}\left[n\left\langle\tau_{j}^{j} \alpha^{\vec{\ell}}, \alpha^{\vec{\ell}}\right\rangle\left\langle\tau_{j}^{j} \alpha^{\vec{r}}, \alpha^{\vec{r}}\right\rangle-\left\langle\tau_{j}^{j} \alpha^{\vec{\ell}}, \alpha^{\vec{\ell}}\right\rangle\left\langle\tau_{p}^{p} \alpha^{\vec{r}}, \alpha^{\vec{r}}\right\rangle\right] d t \\
& =\left(1-\delta_{n-1}^{k}\right) \frac{4 \mu_{2}}{n(n+2)} \lambda_{\vec{\ell}} \lambda_{\vec{r}}\left[n \delta_{j \notin \bar{\ell}} \delta_{j \notin \vec{r}}-\delta_{j \notin \bar{\ell}} \delta_{p \notin \vec{r}}\right] d t \quad\left(\delta_{j \notin \vec{\ell}}=\left\{\begin{array}{l}
1, \text { when } j \notin \vec{\ell}, \\
0, \text { otherwise }
\end{array}\right)\right. \\
& =\left(1-\delta_{n-1}^{k}\right)\left(\frac{4 n \mu_{2}}{n(n+2)} \lambda_{\vec{\ell}} \lambda_{\vec{r}} \cdot \delta_{j \notin \vec{\ell}} \delta_{j \notin \vec{r}} d t-\frac{4(n-k-1)\left(n-\ell-1 \mu_{2}\right)}{n(n+2)} \operatorname{Tr} S^{(k)} \operatorname{Tr} S^{(\ell)} d t\right) .
\end{aligned}
$$

However, by summing on $j$ we see that

$$
\lambda_{\vec{\ell}} \lambda_{\vec{r}} \delta_{j \notin \vec{\ell}} \delta_{j \notin \vec{r}}=\sum_{\substack{m=0 \\\left|\vec{\ell}^{c} \cap \vec{r}^{c}\right|=m}}^{n-\ell-1} m \lambda_{\vec{\ell}} \lambda_{\vec{r}}
$$

which is a symmetric homogeneous polynomial in $\lambda_{1}, \ldots, \lambda_{n-1}$ of degree $k+\ell$ so we seek an expansion for (A.13) of the form

$$
\sum_{j=0}^{(n-k-1) \wedge \ell} d(n,(k, \ell) ; j) \operatorname{Tr} S^{(k+j)} \operatorname{Tr} S^{(\ell-j)} .
$$

The principal term in $\operatorname{Tr} S^{(k+j)} \operatorname{Tr} S^{(\ell-j)}$ (which doesn't appear in $\operatorname{Tr} S^{(k+m)} \operatorname{Tr} S^{(\ell-m)}$ for $\left.m>j\right)$ is

$$
\lambda_{1}^{2} \ldots \lambda_{\ell-j}^{2} \lambda_{\ell-j+1} \ldots \lambda_{k+j} .
$$

In $\sum_{\substack{m-\ell=1 \\|\vec{\ell} \cap \vec{r}|=m}}^{\substack{m=0 \\ \mid \lambda_{\ell}}} \lambda_{\vec{r}}$ this term appears $(n-1-k-j)\left(\begin{array}{c}k-\ell+2 j \\ j\end{array}\right)$ times.

In $\operatorname{Tr} S^{(k+r)} \operatorname{Tr} S^{(\ell-r)}$ for $r<j$ this term appears $\left(\begin{array}{c}k-\ell+2 j \\ j-r\end{array}\right)$ times. Thus in the expansion for $\sum_{\substack{m-\ell-1 \\\left|\vec{\ell}^{c} \cap \vec{r}^{c}\right|=m}}^{n=0} m \lambda_{\vec{\ell}} \lambda_{\vec{r}}$

$$
\begin{aligned}
& d(n,(k, \ell) ; 0)=(n-1-k) \\
& d(n,(k, \ell) ; j)=(n-1-k-j)\left(\begin{array}{c}
k-\ell+2 j \\
j
\end{array}\right)-\sum_{r=0}^{j-1} d(n,(k, \ell) ; r)\left(\begin{array}{c}
k-\ell+2 j \\
j-r
\end{array}\right), j \geq 1 .
\end{aligned}
$$

Putting (A.10), (A.11) and (A.12) together results in

$$
\begin{aligned}
& {\left[\frac{\mu_{2}}{n(n+2)} \operatorname{Tr} S^{(k)} \operatorname{Tr} S^{(\ell)}[(k \ell+2(k+\ell)+4)(n-1)-2(k+2)(n-\ell-1)\right.} \\
& -2(\ell+2)(n-k-1)-4(n-\ell-1)(n-k-1)] \\
& \left.+\left(1-\delta_{n-1}^{k}\right) \frac{4 \mu_{2}}{n(n+2)} \sum_{j=0}^{(n-k-1) \wedge \ell} d(n,(k, \ell) ; j) \operatorname{Tr} S^{(k+j)} \operatorname{Tr} S^{(\ell-j)}\right] d t .
\end{aligned}
$$


The quadratic variation term in $\left\langle d \operatorname{Tr} S^{(k)}, d \operatorname{Tr} S^{(\ell)}\right\rangle, k \geq \ell$, arising from $d B$ is,

$$
\begin{array}{rl}
Q(n,(k, \ell)) d & t \\
& =\left\langle\sum_{i, p=1}^{k} S^{(k-1)}\left(\alpha_{\vec{\ell}_{p}}, \alpha_{\vec{m}_{i}}\right)\left\langle\alpha^{\vec{\ell}}, \alpha^{\vec{m}}\right\rangle\left\langle d B\left(u_{\ell_{p}}, u_{m_{i}}\right), \nu\right\rangle\right. \\
& \left.\sum_{j, q=1}^{\ell} S^{(\ell-1)}\left(\alpha_{\vec{r}_{q}}, \alpha_{\vec{s}_{j}}\right)\left\langle\alpha^{\vec{r}}, \alpha^{\vec{s}}\right\rangle\left\langle d B\left(u_{r_{q}}, u_{s_{j}}\right), \nu\right\rangle\right\rangle\|\alpha\|^{-4} \\
& =C_{1122}^{n n} \sum_{i, p=1}^{k} \sum_{j, q=1}^{\ell} S^{(k-1)}\left(\alpha_{\vec{\ell}_{p}}, \alpha_{\vec{m}_{i}}\right) S^{(\ell-1)}\left(\alpha_{\vec{r}_{q}}, \alpha_{\vec{s}_{j}}\right)\left[\left\langle u_{\ell_{p}}, u_{m_{i}}\right\rangle\left\langle u_{r_{q}}, u_{s_{j}}\right\rangle\right. \\
& \left.+\left\langle u_{\ell_{p}}, u_{r_{q}}\right\rangle\left\langle u_{m_{i}}, u_{s_{j}}\right\rangle+\left\langle u_{\ell_{p}}, u_{s_{j}}\right\rangle\left\langle u_{r_{q}}, u_{m_{i}}\right\rangle\right]\left\langle\alpha^{\vec{\ell}}, \alpha^{\vec{m}}\right\rangle\left\langle\alpha^{\vec{r}}, \alpha^{\vec{s}}\right\rangle\|\alpha\|^{-4} d t
\end{array}
$$

and taking advantage of the invariance of this expression under a change of basis, we take $u_{i}$ to be the $i$ th unit principal direction of curvature. Then

$$
\begin{aligned}
& S^{(k-1)}\left(\alpha_{\vec{\ell}_{p}}, \alpha_{\vec{m}_{i}}\right)\left\langle\alpha^{\vec{\ell}}, \alpha^{\vec{m}}\right\rangle=\lambda_{\vec{\ell}_{p}} \delta_{\vec{m}}^{\vec{\ell}} \delta_{p}^{i} \\
& S^{\ell-1}\left(\alpha_{\vec{r}_{q}}, \alpha_{\vec{s}_{j}}\right)\left\langle\alpha^{\vec{r}}, \alpha^{\vec{s}}\right\rangle=\lambda_{\vec{r}_{q}} \delta_{\vec{s}}^{\vec{r}} \delta_{q}^{j} \\
& {\left[\left\langle u_{\ell_{p}}, u_{m_{i}}\right\rangle\left\langle u_{r_{q}}, u_{s_{j}}\right\rangle+\left\langle u_{\ell_{p}}, u_{r_{q}}\right\rangle\left\langle u_{m_{i}}, u_{s_{j}}\right\rangle+\left\langle u_{\ell_{p}}, u_{s_{j}}\right\rangle\left\langle u_{r_{q}}, u_{m_{i}}\right\rangle\right] \delta_{\vec{m}} \delta_{\vec{s}}^{\vec{r}} } \\
&=\left(\delta_{m_{i}}^{\ell_{p}} \delta_{s_{j}}^{r_{q}}+\delta_{r_{q}}^{\ell_{p}} \delta_{s_{j}}^{m_{i}}+\delta_{s_{j}}^{\ell_{p}} \delta_{m_{i}}^{r_{q}}\right) \delta_{\vec{m}} \delta_{\vec{s}}^{\vec{r}} .
\end{aligned}
$$

Thus,

$$
\begin{aligned}
Q(n,(k, \ell)) & =C_{1122}^{n n} \sum_{p=1}^{k} \sum_{q=1}^{\ell} \lambda_{\vec{\ell}_{p}} \lambda_{\vec{r}_{q}}\left(1+2 \delta_{r_{q}}^{\ell_{p}}\right) d t \\
& =C_{1122}^{n n}\left(3 \sum_{p=1}^{k} \sum_{\substack{q=1 \\
\ell_{p}=r_{q}}}^{\ell} \lambda_{\vec{\ell}_{p}} \lambda_{\vec{r}_{q}}+\sum_{p=1}^{k} \sum_{\substack{q=1 \\
\ell_{p} \neq r_{q}}}^{\ell} \lambda_{\vec{\ell}_{p}} \lambda_{\vec{r}_{q}}\right) .
\end{aligned}
$$

Since $Q(n,(k, \ell))$ is a symmetric homogeneous polynomial of degree $k+\ell-2$ in $\lambda_{1}, \ldots, \lambda_{n-1}$, it may be written

$$
Q(n,(k, \ell))=C_{1122}^{n n} \sum_{j=1}^{(n-k) \wedge(\ell-1)} a(n,(k, \ell) ; j) \operatorname{Tr} S^{(k+j-1)} \operatorname{Tr} S^{(\ell-j-1)}
$$

We now employ some elementary counting arguments to compute the coefficients $a(n,(k, \ell) ; j)$.

In $\operatorname{Tr} S^{(k+j-1)} \operatorname{Tr} S^{(\ell-j-1)}$ the term

$$
\lambda_{1}^{2} \ldots \lambda_{\ell-j-1}^{2} \lambda_{\ell-j} \ldots \lambda_{k+j-1} \quad \text { appears }
$$


but no such term (with $\ell-j-1$ squares) appears in $\operatorname{Tr} S^{(k+m-1)} \operatorname{Tr} S^{(\ell-m-1)}$ for $m>j$. However, if $r<j$,

$$
\lambda_{1}^{2} \ldots \lambda_{\ell-j-1}^{2} \lambda_{\ell-j} \ldots \lambda_{k+j-1} \quad \text { appears }
$$

$\left(\begin{array}{c}k-\ell+2 j \\ j-r\end{array}\right)$ times in $\operatorname{Tr} S^{(k+r-1)} \operatorname{Tr} S^{(\ell-r-1)}$.

We now count the number of appearances of $(\mathrm{A} .16)$ in $Q(n,(k, \ell))$. From $3 \sum_{p=1}^{k} \sum_{\substack{q=1 \\ \ell_{p}=r_{q}}}^{\ell} \lambda_{\vec{\ell}_{p}} \lambda_{\vec{r}_{q}}$ we must fill in the empty spaces in $\lambda_{\vec{r}}$ and $\lambda_{\vec{\ell}}$

$$
\begin{aligned}
& \lambda_{\vec{r}}=\lambda_{1} \ldots \lambda_{\ell-j-1}-\cdots- \\
& \lambda_{\vec{\ell}}=\lambda_{1} \ldots \lambda_{\ell-j-1}-\cdots-{ }_{\ell} \cdots-_{k}
\end{aligned}
$$

with the terms $\lambda_{\ell-j}, \ldots, \lambda_{k+j-1}, \lambda_{x}, \lambda_{x}$, where $x=\ell_{p}=r_{q}$. Notice $\lambda_{t}$ appears before $\lambda_{u}$ only if $t<u$. One $\lambda_{x}$ must go in $\lambda_{\vec{r}}$ the other in $\lambda_{\vec{\ell}}$. If the order is not to be violated, this forces $x>k-1+j$ and $\lambda_{x}$ must end each of the terms $\lambda_{\vec{r}}$ and $\lambda_{\vec{\ell}}$. There are $(n-1)-(k-1+j)=n-k-j$ such choices for $x$. This leaves

$$
\left(\begin{array}{c}
k-1+j-(\ell-j-1) \\
j
\end{array}\right)=\left(\begin{array}{c}
k-\ell+2 j \\
j
\end{array}\right)
$$

remaining possibilities for filling in the empty spaces in (A.18) in $\lambda_{\vec{r}}$ and $\lambda_{\vec{\ell}}$. Note that if we interpret $\left(\begin{array}{l}0 \\ 0\end{array}\right)=1$ this is still correct for $k=\ell$ and $j=0$. Thus

$$
3 \sum_{p=1}^{k} \sum_{\substack{q=1 \\
\ell_{p}=r_{q}}}^{\ell} \lambda_{\vec{\ell}_{p}} \lambda_{\vec{r}_{q}} \text { has } 3(n-k-j)\left(\begin{array}{c}
k-\ell+2 j \\
j
\end{array}\right)
$$

terms of the form $\lambda_{1}^{2} \ldots \lambda_{\ell-j-1}^{2} \lambda_{\ell-j} \ldots \lambda_{k+j-1}$.

$$
\begin{aligned}
\operatorname{In} \sum_{p=1}^{k} \sum_{\substack{q=1 \\
\ell_{p} \neq r_{q}}}^{\ell} \lambda_{\vec{\ell}_{p}} \lambda_{\vec{r}_{q}} \text { we must fill in the blanks of } \\
\\
\lambda_{\vec{r}}=\lambda_{1} \ldots \lambda_{\ell-j-1}-\cdots- \\
\lambda_{\vec{\ell}}=\lambda_{1} \ldots \lambda_{\ell-j-1}-\cdots-\ell \cdots-k
\end{aligned}
$$

with $\lambda_{\ell-j}, \ldots, \lambda_{k+j-1}, \lambda_{x}, \lambda_{y}$ with $x \neq y, \lambda_{x}$ going into $\lambda_{\vec{r}}, \lambda_{y}$ going into $\lambda_{\vec{\ell}}$.

If $\ell-j \leq x, y \leq k+j-1$, then $\lambda_{x}$ and $\lambda_{y}$ appear on the list $\lambda_{\ell-j}, \ldots, \lambda_{k+j-1}$. Since identical terms can't appear twice in $\lambda_{\vec{r}}$ or $\lambda_{\vec{\ell}}$ the twin of $\lambda_{x}$ must go in $\lambda_{\vec{\ell}}$, the twin of $\lambda_{y}$ must go in $\lambda_{\vec{r}}$. There are thus $\left(\begin{array}{c}k-\ell+2 j-2 \\ j-1\end{array}\right)$ ways to fill in the spaces once $\lambda_{x}$ and $\lambda_{y}$ are given with $\ell-j \leq x, y \leq k+j-1$. There are $(k+j-1-(\ell-$ 
$j-1))(k+j-1-(\ell-j-1)-1)$ choices for the ordered pair $(x, y)$. Notice that when $j=0$ there are no such terms. Thus

$$
\begin{aligned}
& \ell-j \leq x, y \leq k+j-1 \text { accounts for } \\
& (k-\ell+2 j)(k-\ell+2 j-1)\left(\begin{array}{c}
k-\ell+2 j-2 \\
j-1
\end{array}\right)
\end{aligned}
$$

terms of the form $\lambda_{1}^{2} \ldots \lambda_{\ell-j-1}^{2} \lambda_{\ell-j} \ldots \lambda_{k+j-1}$ in $Q\left(n,(k, \ell)\right.$ ) (interpreting $\left(\begin{array}{c}z \\ -1\end{array}\right)=$ 0.)

If $\ell-j \leq x \leq k+j-1<y \leq n-1$, then $\lambda_{y}$ must terminate the sequence $\lambda_{\vec{\ell}}$ and the twin of $\lambda_{x}$ must go into $\lambda_{\vec{\ell}}$. There are then $\left(\begin{array}{c}k-\ell+2 j-1 \\ j\end{array}\right)$ ways to fill in the spaces in (A.18) once such a pair $(x, y)$ is given. We interpret this expression as 0 when $k=\ell$ and $j=0$. There are $(k-\ell+2 j)(n-k-j)$ such pairs so we have

$$
\begin{aligned}
& \text { corresponding to } \ell-j \leq x \leq k+j-1<x \leq n-1 \\
& \text { there are }(k-\ell+2 j)(n-k-j)\left(\begin{array}{c}
k-\ell+2 j-1 \\
j
\end{array}\right) \\
& \text { terms of the form } \lambda_{1}^{2} \cdots \lambda_{\ell-j-1}^{2} \lambda_{\ell-j} \cdots \lambda_{k+j-1} \text { in } \\
& Q(n,(k, \ell)) \text {. }
\end{aligned}
$$

If $\ell-j \leq y \leq k+j-1<x \leq n-1$, then the twin of $\lambda_{y}$ must go in $\lambda_{\vec{r}}$. When $j=0$, this is impossible. There are thus $\left(\begin{array}{c}k-\ell+2 j-1 \\ j-1\end{array}\right)$ ways to fill the spaces in $(\mathrm{A} .18)$ once $(x, y)$ is given and $(k-\ell+2 j)(n-k-j)$ such pairs so

corresponding to $\ell-j \leq y \leq k+j-1<x \leq n-1$,

there are $(k-\ell+2 j)(n-k-j)\left(\begin{array}{c}k-\ell+2 j-1 \\ j-1\end{array}\right)$

terms of the form $\lambda_{1}^{2} \cdots \lambda_{\ell-j-1}^{2} \lambda_{\ell-j} \cdots \lambda_{k+j-1}$ in

$$
Q(n,(k, \ell)) \text {, for } j \geq 0 \text {, (interpreting }\left(\begin{array}{c}
z \\
-1
\end{array}\right)=0 \text {.) }
$$

Finally, when $k+j-1<x, y \leq n-1$, there are $\left(\begin{array}{c}k-\ell+2 j \\ j\end{array}\right)$ ways to fill in the spaces in (A.18) once $(x, y)$ is given and $(n-k-j)(n-k-j-1)$ such pairs $(x, y)$. Thus

corresponding to $k+j-1<x, y \leq n-1$, there are $(n-k-j)(n-k-j-1)\left(\begin{array}{c}k-\ell+2 j \\ j\end{array}\right)$ terms of the form $\lambda_{1}^{2} \cdots \lambda_{\ell-j-1}^{2} \lambda_{\ell-j} \cdots \lambda_{k+j-1}$ in $Q(n,(k, \ell))$, interpreting $\left(\begin{array}{l}0 \\ 0\end{array}\right)=1$. 
Totalling (A.18)-(A.23), we see

$$
\begin{aligned}
Q(n, k) & \text { has } \\
b(n,(k, \ell) ; j) & =3(n-k-j)\left(\begin{array}{c}
k-\ell+2 j \\
j
\end{array}\right) \\
& +(k-\ell+2 j)(k-\ell+2 j-1)\left(\begin{array}{c}
k-\ell+2 j-2 \\
j-1
\end{array}\right) \\
& +(k-\ell+2 j)(n-k-j)\left(\begin{array}{c}
k-\ell+2 j-1 \\
j
\end{array}\right) \\
& +(k-\ell+2 j)(n-k-j)\left(\begin{array}{c}
k-\ell+2 j-1 \\
j-1
\end{array}\right) \\
& +(n-k-j)(n-k-j-1)\left(\begin{array}{c}
k-\ell+2 j \\
j
\end{array}\right)
\end{aligned}
$$

terms with exactly $\ell-j-1$ squares. Notice that we interpret $\left(\begin{array}{c}z \\ -1\end{array}\right)=0,\left(\begin{array}{c}-1 \\ 0\end{array}\right)=0$, $\left(\begin{array}{l}0 \\ 0\end{array}\right)=1$. Thus, $b(n,(k, \ell) ; 0)=(n-k)(n-k-2)$. Upon decomposing $Q(n,(k, \ell))$ into sums of expressions with exactly $\ell-j-1$ squares summed over $j$, we are lead to, using (A.16) and (A.23),

$$
Q(n, k)=C_{1122}^{n n} \sum_{j=0}^{(n-k) \wedge(\ell-1)} a(n,(k, \ell) ; j) \operatorname{Tr} S^{(k+j-1)} \operatorname{Tr} S^{(\ell-j-1)}
$$

where

$$
\begin{aligned}
& a(n,(k, \ell) ; j)=b(n,(k, \ell) ; j)-\sum_{m=0}^{j-1}\left(\begin{array}{c}
k-\ell+2 j \\
j-m
\end{array}\right) a(n,(k, \ell) ; m) \\
& a(n,(k, \ell) ; 0)=b(n,(k, \ell) ; 0)=(n-k)(n-\ell+2) .
\end{aligned}
$$

From Theorems A.2 and A.3, we get immediately

Theorem A.4. Suppose $\Phi_{t}$ is the isotropic, measure preserving flow on $\mathbb{R}^{n}$ given by (1.3). Let $M$ be a smooth hypersurface in $\mathbb{R}^{n}$ with $x \in M$. If $\left(\lambda_{1}(t), \ldots, \lambda_{n-1}(t)\right)$ are the principal curvatures of $\Phi_{t}(M)$ at $\Phi_{t}(x)$, then

$$
\operatorname{Tr} S^{(k)}=\sum_{\vec{\ell} \in I_{k}} \lambda_{\vec{\ell}}
$$

and $\left(\operatorname{Tr} S^{(1)}, \operatorname{Tr} S^{(2)}, \ldots, \operatorname{Tr} S^{(n-1)}\right)$ is an $(n-1)$-dimensional diffusion. The 
generator is given by

$$
\begin{aligned}
L f\left(x_{1}, \ldots, x_{n-1}\right)= & \sum_{1 \leq \ell \leq k \leq n-1}\left(\frac{1}{2}+\frac{1}{2}\left(1-\delta_{\ell}^{k}\right)\right) \\
& \left\{\frac{\mu_{2}}{n(n+2)}[((k \ell+2(k+\ell)+4)(n-1)\right. \\
- & 2(k+2)(n-\ell-1)-2(\ell+2)(n-k-1) \\
& -4(n-\ell-1)(n-k-1)) x_{k} x_{\ell} \\
+ & \left.\left(1-\delta_{n-1}^{k}\right) 4 n \sum_{j=0}^{(n-k-1) \wedge \ell} d(n,(k, \ell) ; j) x_{k+j} x_{\ell-j}\right] \\
+ & \left.C_{1122}^{n n} \sum_{j=0}^{(n-k) \wedge(\ell-1)} a(n,(k, \ell) ; j) x_{k+j-1} x_{\ell-j-1}\right\} \frac{\partial^{2} f}{\partial x_{k} \partial x_{\ell}} \\
+ & \sum_{k=1}^{n-1} \frac{(n+1) k(n-k)) \mu_{2}}{2 n(n+2)} x_{k} \frac{\partial f}{\partial x_{k}}
\end{aligned}
$$

where, for $k \geq \ell$

$$
\begin{aligned}
d(n,(k, \ell) ; 0) & =(n-1-k) \\
d(n,(k, \ell) ; j) & =(n-1-k-j)\left(\begin{array}{c}
k-\ell+2 j \\
j
\end{array}\right)-\sum_{r=0}^{j-1} d(n,(k, \ell) ; r)\left(\begin{array}{c}
k-\ell+2 j \\
j-r
\end{array}\right), j \geq 1 \\
b(n,(k, \ell) ; j) & =3(n-k-j)\left(\begin{array}{c}
k-\ell+2 j \\
j
\end{array}\right)+(k-\ell+2 j)(k-\ell+2 j-1)\left(\begin{array}{c}
k-\ell+2 j-2 \\
j-1
\end{array}\right) \\
& +(k-\ell+2 j)(n-k-j)\left[\left(\begin{array}{c}
k-\ell+2 j-1 \\
j
\end{array}\right)+\left(\begin{array}{c}
k-\ell+2 j-1 \\
j-1
\end{array}\right)\right] \\
& +(n-k-j)(n-k-j-1)\left(\begin{array}{c}
k-\ell+2 j \\
j
\end{array}\right) ; j \geq 0, \text { with }\left(\begin{array}{c}
z \\
-1
\end{array}\right)=\left(\begin{array}{c}
-1 \\
0
\end{array}\right),\left(\begin{array}{l}
0 \\
0
\end{array}\right)=1, \\
a(n,(k, \ell) ; 0) & =b(n,(k, \ell) ; 0)=(n-k)(n-\ell+2), \\
a(n,(k, \ell) ; j) & =b(n,(k, \ell) ; j)-\sum_{r=0}^{j-1} a(n,(k, \ell) ; r)\left(\begin{array}{c}
k-\ell+2 j \\
j-r
\end{array}\right), j>0 \\
C_{1122}^{n n} & =\frac{(n+3) \mu_{4}}{n(n+2)(n+4)},
\end{aligned}
$$

and $x_{-1}=x_{0}=1$. 


\section{Remarks.}

(1) Straightforward computations show that for $n=3$

$$
\begin{gathered}
k=\ell=1 ; \quad \begin{array}{l}
d(3,(1,1) ; 0)=1 \\
d(3,(1,1) ; 1)=-2 \\
a(3,(1,1) ; 0)=8
\end{array} \\
k=2, \ell=1 ; \quad a(3,(2,1) ; 0)=4 \\
k=2, \ell=2 ; \quad a(3,(2,2) ; 0)=3 \\
a(3,(2,2) ; 1)=-4
\end{gathered}
$$

Thus, when $M$ is a hypersurface in $\mathbb{R}^{3}$, the pair $\left(\operatorname{Tr} S^{(1)}, \operatorname{Tr} S^{(2)}\right)$ is a diffusion with generator

$$
\begin{aligned}
L f(\kappa, m) & =\frac{1}{2}\left(\frac{\mu_{2}}{15}\left(14 \kappa^{2}-24 m\right)+\frac{16 \mu_{4}}{35}\right) \frac{\partial^{2} f}{\partial \kappa^{2}}(\kappa, m) \\
& +\left(\frac{16 \mu_{2}}{15} \kappa m+\frac{8 \mu_{4}}{35} \kappa\right) \frac{\partial^{2} f}{\partial \kappa \partial m}(\kappa, m) \\
& +\frac{1}{2}\left(\frac{32 \mu_{2}}{15} m^{2}+\frac{2 \mu_{4}}{35}\left(3 \kappa^{2}-4 m\right)\right) \frac{\partial^{2} f}{\partial m^{2}}(\kappa, m) \\
& +\frac{4 \mu_{2}}{15} \kappa \frac{\partial f}{\partial \kappa}(\kappa, m)+\frac{4 \mu_{2}}{15} m m \frac{\partial f}{\partial m}(\kappa, m)
\end{aligned}
$$

which agrees with Theorem 2.9 .

(2) For $n=4, a=\frac{\mu_{2}}{24}, b=C_{1122}^{44}=\frac{7 \mu_{4}}{192}$,

$$
\begin{aligned}
L f(x, y, z) & =\frac{1}{2}\left(a\left(19 x^{2}-32 y\right)+15 b\right) \frac{\partial^{2} f}{\partial x^{2}} \\
& +\frac{1}{2}\left(a\left(44 y^{2}-32 x z\right)+b\left(8 x^{2}-4 y\right)\right) \frac{\partial^{2} f}{\partial y^{2}} \\
& +\frac{1}{2}\left(a 75 z^{2}+b\left(3 y^{2}-4 x z\right)\right) \frac{\partial^{2} f}{\partial z^{2}} \\
& +(a(22 x y-48 z)+b 10 x) \frac{\partial^{2} f}{\partial x \partial y}+(a 25 x z+b 5 y) \frac{\partial^{2} f}{\partial x \partial z} \\
& +(a 50 y z+b(4 x y-6 z)) \frac{\partial^{2} f}{\partial y \partial z} \\
& +a \frac{15}{2} x \frac{\partial f}{\partial x}+a 10 y \frac{\partial f}{\partial y}+a \frac{15}{2} z \frac{\partial f}{\partial z} .
\end{aligned}
$$

(3) For any $n$, no proper subset of $\left\{\operatorname{Tr} S^{(1)}, \ldots, \operatorname{Tr} S^{(n-1)}\right\}$ can be a diffusion. 
(4) The authors intend to resolve such issues as transience or recurrence for higher dimensional cases.

(5) Since $x=\lambda_{1}+\lambda_{2}+\lambda_{3}, y=\lambda_{1} \lambda_{2}+\lambda_{1} \lambda_{3}+\lambda_{2} \lambda_{3}, z=\lambda_{1} \lambda_{2} \lambda_{3}$, Newton's inequalities, Hardy-Littlewood- Polya (1973), imply $x^{2} \geq 3 y, y^{2} \geq 3 x z$ with equality if and only if $\lambda_{1}=\lambda_{2}=\lambda_{3}$. As in the case $n=3$, the diffusion matrix degenerates on $\lambda_{1}=\lambda_{2}=\lambda_{3}$ and one expects that the process will not reach this set at positive times. 